\title{
ARTISANALLY LANDED ELASMOBRANCHS ALONG THE COAST OF RIO DE JANEIRO, BRAZIL
}

Nathan Lagares Franco Araujo ${ }^{1,2}$, Catarina Amorim Lopes ${ }^{1,2,3}$, Vanessa Bettcher Brito ${ }^{2,3}$, Luciano Neves dos Santos ${ }^{2}$, Márcio Luiz Vargas Barbosa-Filho ${ }^{4}$, César Rogério Leal do Amaral ${ }^{5}$, Salvatore Siciliano $^{6,7}$, Rachel Ann Hauser-Davis ${ }^{1,7 *}$ * corresponding author: rachel.hauser.davis@gmail.com; rachel.davis@ ioc.fiocruz.br

${ }^{1}$ Laboratório de Avaliação e Promoção da Saúde Ambiental, Instituto Oswaldo Cruz/Fiocruz, Av. Brasil, 4.365, Manguinhos, Rio de Janeiro, RJ, 21040-360, Brazil.

${ }^{2}$ Laboratório de Ictiologia Teórica e Aplicada, Instituto de Biociências, Universidade Federal do Estado do Rio de Janeiro, Avenida Pasteur, 458, Urca, Rio de Janeiro, RJ, 22290-255, Brazil.

${ }^{3}$ Programa de Pós-graduação em Ecologia e Evolução, Universidade Estadual do Rio de Janeiro, Rua São Francisco Xavier, 524, Maracanã, 20550-900, Rio de Janeiro, RJ, Brazil

${ }^{4}$ Programa de Pós-graduação em Etnobiologia e Conservação da Natureza, Universidade Federal Rural de Pernambuco, Campus Dois Irmãos, Recife, 52171-900, PE, Brazil

${ }^{5}$ Laboratório de Diagnósticos por DNA, Instituto de Biologia Roberto Alcantara Gomes, Universidade do Estado do Rio de Janeiro, São Francisco Xavier, 524, Maracanã, Rio de Janeiro, RJ, 20550-013, Brazil

${ }^{6}$ Laboratório de Biodiversidade, Instituto Oswaldo Cruz, Fiocruz, Pavilhão Mourisco Sala 18, Manguinhos, Rio de Janeiro, RJ 21040-900 Brazil

${ }^{7}$ Grupo de Estudos de Mamíferos Marinhos da Região dos Lagos (GEMM-Lagos), Rua São José 1.260, Praia Seca, Araruama, RJ 28970-000 Brazil

\section{RESUMO}

Considerando a lacuna de conhecimento em relação aos desembarques de espécies de elasmobrânquios, este estudo teve como objetivo relatar as capturas de elasmobrânquios através da pesca artesanal para obter dados de base a esse respeito. As amostragens foram realizadas de 2016 a 2019 em três colônias de pesca artesanal localizadas no Rio de Janeiro, Tamoios, em Cabo Frio, Itaipu, em Niterói e Copacabana, na região metropolitana do Rio de Janeiro. Um total de vinte e três espécies de doze famílias foi identificado em todos os locais da amostra, incluindo 10 tubarões e 12 raias. Embora Tamoios, em Cabo Frio, sofra os efeitos de um fenômeno de ressurgência, a maior riqueza relativa a elasmobrânquios oceânicas foi observada em Copacabana, que faz parte da Baía de Guanabara, reforçando a biodiversidade significativa dessa baía e a importância de sua recuperação para estratégias de manejo e conservação de elasmobrânquios. Além disso, muitos espécimes pertencentes a diferentes espécies amostradas neste local eram juvenis, como tubarões martelo (Sphyrna lewini), tigre (Galeocerdo cuvier), mako Isurus oxyrinchus), seis fêmeas de cações frango (Rhizoprionodon lalandii) contendo embriões ou oocitos e três fêmeas de tubarão-rotador (Carcharinus brevipinna) com oócitos maduros, indicando um possível local estratégico de reprodução e assentamento juvenil para várias espécies desembarcadas. A presença de muitas espécies apresentando diferentes graus de vulnerabilidade em relação à conservação e endemismo, especialmente para o Atlântico Sul, reforça a importância desse tipo de avaliação. Além disso, quatro de seis espécies-chave de elasmobrânquios, ou seja, espécies que apresentam um impacto extremamente alto em um ecossistema específico em relação à sua população e críticas para a estrutura e função gerais de um ecossistema, anteriormente observadas na costa sudeste do Brasil, são relatadas aqui, a saber Galeocerdo cuvier, Sphyrna lewini, S. zygaena e Zapteryx brevirostris, indicando ainda a importância de futuras avaliações sobre o monitoramento da pesca de elasmobrânquias no Brasil.

Palavras-chave: Biodiversidade; tubarões e raias; pesca artesanal.

\section{ABSTRACT}

Considering the knowledge gap regarding elasmobranch species landings, this study aimed to report elasmobranch catches from artisanal fisheries to obtain baseline data in this regard. Samplings were carried out from 2016 to 2019 at three artisanal fishing colonies located in Rio de Janeiro, Tamoios, in Cabo Frio, Itaipu, in Niterói, and Copacabana, in the metropolitan region of Rio de Janeiro. A total of twentythree species from twelve families were identified at all sample sites, comprising 10 sharks and 12 rays. Although Tamoios, in Cabo Frio, is home to an upwelling phenomenon, the highest richness concerning oceanic elasmobranchs was observed at Copacabana, which is a part of Guanabara Bay, reinforcing the significant biodiversity of this bay and the importance of its recovery for elasmobranch management and conservation strategies. In addition, many specimens belonging to different species sampled at this site were juvenile, such as hammerhead sharks (Sphyrna lewini), tiger sharks (Galeocerdo cuvier), mako sharks (Isurus oxyrinchus), six sharpnose sharks (Rhizoprionodon lalandii) females containing embryos and/or oocytes and three spinner shark females (Carcharinus brevipinna) containing mature oocytes, indicating a possible strategic reproduction and juvenile settlement site for several of the landed species. The presence of many species presenting different vulnerability degrees regarding conservation and endemism, especially for the South Atlantic, reinforces the importance of this type of assessment. In addition, four of six keystone elasmobranch species, i.e. species presenting an extremely high impact on a particular ecosystem relative to its population and critical for the overall structure and function of an ecosystem, previously observed throughout the Southeastern coast of Brazil are reported herein, namely Galeocerdo cuvier, Sphyrna lewini, S. zygaena and Zapteryx brevirostris, further indicating the importance of future assessments concerning elasmobranch fisheries monitoring in Brazil. 
Key-words: Biodiversity; sharks and rays; artisanal fisheries.

\section{INTRODUCTION}

Brazil is home to approximately one million registered artisanal fishers, which contribute to over 50\% of the total fish produced in the country (Vasconcellos et al. 2007). However, data concerning artisanal fisheries are poor (Kaliloski \& Vasconcellos 2012; Previero \& Gasalla 2018), making it difficult to identify and assess the effectiveness of conservation management actions (Costa et al., 2018). Elasmobranchs in particular are extremely vulnerable to artisanal fishery (Lack \& Sant 2009; Bornatowski et al. 2014), either targeted or captured as bycatch (Molina \& Cooke 2012; Ferrette $e t$ al. 2019), as they are long lived organisms displaying slow growth rates, delayed maturation, long gestation, and small litters (Stevens et al. 2000; Dulvy et al. 2014). Approximately $25 \%$ of this class has been reported as threatened with extinction by the International Union for Conservation of Nature (IUCN) (Dulvy et al. 2014) and, $47 \%$ of these are classified by the IUCN as data deficient, indicating absence of minimal information to be evaluated (Mace et al. 2008; Dulvy et al. 2014).

In many countries, shark and ray meat are viewed as a low-quality food item and, thus, marketed under generic designations not directly linked to these animals, in order to overcome consumer resistance (Vannuccini 1999; Bornatowski et al. 2013, 2015; Dent $\&$ Clarke 2015). This is the case in Brazil, where shark and ray meat are sold as cação fillets (Bornatowski et al. 2018). The lack of consumer knowledge regarding cação meat and what it really consists in has increasingly led to overfishing due to attractive shark and ray meat prices (Bornatowski et al. 2018) and is of significant ecological concern, as these animals play an important role in maintaining ecosystem equilibrium and health (Heupel et al. 2014). In this scenario, due to both targeted and non-targeted fisheries, Brazilian fisheries have reached critical levels for several elasmobranch species (Bornatowski et al. 2018) and 33\% of all elasmobranchs are categorized as threatened, while $36 \%$ are considered data deficient (Instituto Chico Mendes de Conservação da Biodiversidade 2018), overcoming the global rate of threatened species. Most pelagic shark populations are currently depleted in Brazil while coastal species data are very poor (Bornatowski et al. 2018).

A constant that determines the difficulty of social and ecological fishing activity management in Brazil is the lack of specific monitoring for each fishing sector, both large and small (artisanal) scale, in the long term, as, although fishery statistics are achieved regionally by some states, most of the country lacks this specificity. However, effective governance between conservation and fisheries can only be effective by filling in the gaps in fisheries data (Kolding et al., 2010). In Brazil, the latest version of the National Fisheries and Aquaculture Statistical Bulletin (Instituto Chico Mendes de Conservação da Biodiversidade, 2011) illustrates the main problem faced by sector managers, of data discontinuity, since this government document has not been updated for eight years. At the state level, the Rio de Janeiro Institute of Fisheries Foundation issues an annual report on Fisheries and Aquaculture where, in addition to unspecific fish categorization, a combination of large and scale small data is noted, making it very difficult, for example, to discern which species are vulnerable to what types of fisheries, leading to significant data deficiency, especially with regard to artisanal fishing.

However, no recent evaluation of artisanally landed sharks and rays are available for many artisanal fisheries regions, such as those located in the metropolitan region of Rio de Janeiro, and the Região dos Lagos region, which comprises over 1500 registered artisanal fishers from at least eight cities (Saquarema, Maricá, Araruama, Iguaba Grande, São Pedro da Aldeia, Cabo Frio, Búzios and Arraial do Cabo) (FIPERJ 2015).

In this context, this study aimed to assess artisanally landed sharks and rays along the coast of Rio de Janeiro, RJ, Brazil through fisheries monitoring, fisher interviews and photographs taken by the fishers and the researchers.

\section{MATERIAL AND METHODS}

The state of Rio de Janeiro ( $22^{\circ} 54^{\prime} 13^{\prime \prime} \mathrm{S}, 43^{\circ}$ $\left.12^{\prime} 35^{\prime \prime} \mathrm{W}\right)$, is located in southeastern Brazil, occupying the $4^{\text {th }}$ place in terms of economy and $3^{\text {rd }}$ in population size in the national ranking (IBGE, 2019), extremely important in a socio-economic context.

A total of 28 artisanal fisher colonies are distributed throughout the state of Rio de Janeiro, from São Francisco do Itabapoana to Paraty (FIPERJ, 2019), with the main fishing ports located at Niterói, São Gonçalo, Cabo Frio and Angra dos Reis.

Samplings were carried out at two artisanal fishing unions and one fisher association located in Rio de Janeiro, namely the Z-13 fishing colony/union, in Copacabana (22 59 ' 10 "S, $43^{\circ} 11^{\prime}$ ' $19^{\prime}$ ” W), in the metropolitan Rio de Janeiro area, the Associação de Pesca de Tamoios (Tamoios Fishers Association), located in the Pontal de Santo Antônio, in Cabo Frio $\left(22^{\circ} 35^{\prime} 55.0^{\prime \prime} \mathrm{S}, 41^{\circ} 59^{\prime} 40.9^{\prime}\right.$ ' W), on the Southeastern coast of the state, and the Z-7 fishing colony/union, at 
Itaipu (2253'14'’S, 4322'48”W), in Niterói (Figure 1).

Located in the central region of the state, artisanal fisher colonies Z-13 and Z-7, at Copacabana and Itaipu, respectively, operate in fishing areas that undergo strong influences from Guanabara Bay, an eutrophic estuarine environment, mainly in the regions most associated with oceanic waters. On the other hand, the Associação de Pesca de Tamoios, in Cabo Frio is located in an area characterized by a significant upwelling phenomenon, where deep South Atlantic Central Waters rise continuously, resulting in a high abundance of nectonic species, making this area one of the most productive fishing areas in the state of Rio de Janeiro (Valentin, 2001).

No standardized sampling effort was carried out in this study, due to logistic reasons. All specimens were caught within the fishing spectrum of the respective sites, no further that $15 \mathrm{~km}$ from the fishing colonies.

Samples from the Z-7 Itaipu colony, which consists of 130 associated fishers, were obtained from January to July 2016, comprising 10 visits at random. Initial visits by the researchers to this colony enabled the development of both a close relation with the fishers and elasmobranch landings, so, in the absence of the research team, fishers would send photographs of elasmobranchs whenever these animals were landed. Elasmobranch landing data for Itaipu were obtained from Brito (2016). Concerning Tamoios, periodic visits were made every three months to the Associação de Pesca de Tamoios, from 2017 to 2018. At Tamoios, landing assessments were always performed by the research team, through sample collections and photographic records, without the aid of local fishermen.
Data collection at the Z-13 colony was carried out from September 2018 to September 2019. Observations and species identification through the scientific literature (Gomes et al. 2010) were performed through frequent visits to the colony, of at least 3 times a week, according to sea conditions, obtaining photographic records and/or tissue samples of the sampled animals. In the absence of the research team, fishers would send photographs of elasmobranchs whenever these animals were landed.

The same fishing gear, gillnets, was used in all three regions. Only the amount of cloths and mesh size differ among the fishing colonies. All nets had an average height of two meters. At Copacabana, $50 \mathrm{~mm}$ mesh gillnets are used for bottom and mid-column fishing, with about 8 cloths per boat, resulting in about 400 to $500 \mathrm{~m}$ nets for each boat. At Tamoios, a mesh size between 40 and $45 \mathrm{~mm}$ is used, with varying amounts of cloths according to boat size, of about $100 \mathrm{~m}$ each, reaching up to 10 cloths in a single boat. At Itaipu, gillnets with mesh sizes varying from 40 to $80 \mathrm{~mm}$ are used for mid-column and bottom fishing. No information on net lengths are available for this colony. All elasmobranchs were captured as by-catch, due to the non-selectivity of the fishing methodology, even at different depths.

\section{RESULTS}

A total of twenty-three species from twelve families were identified at all sample sites. Data concerning the Order, Family, popular names, global and national Conservation States, and geographic distribution of the artisanally landed elasmobranchs

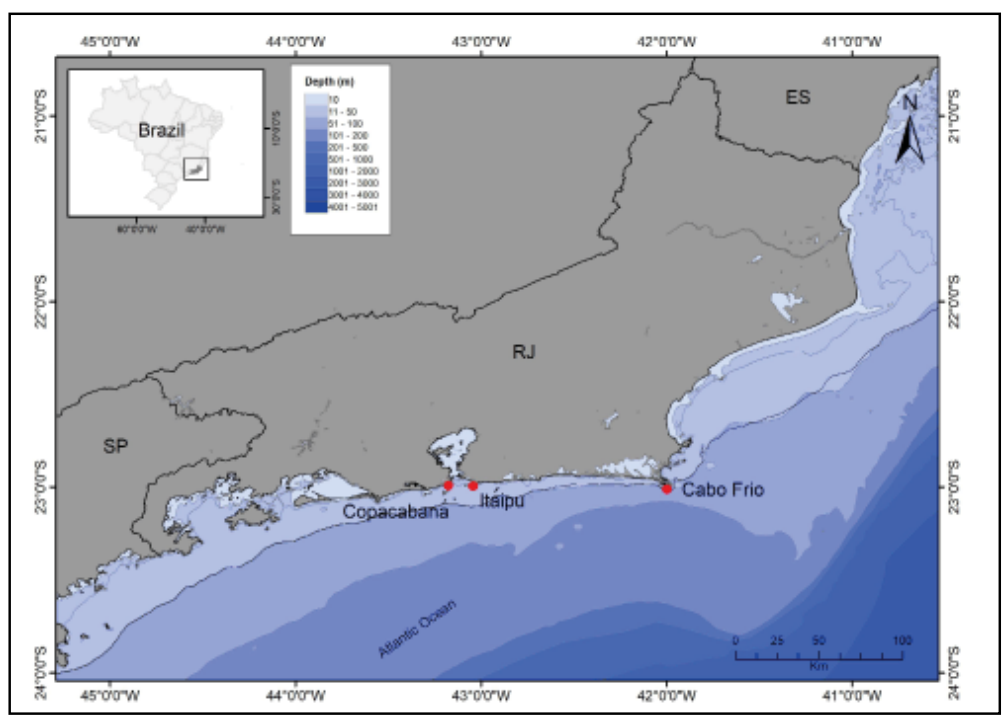

Figure 1. Map of the three artisanal fisher colony locations assessed in the present study concerning elasmobranch by-catches. 


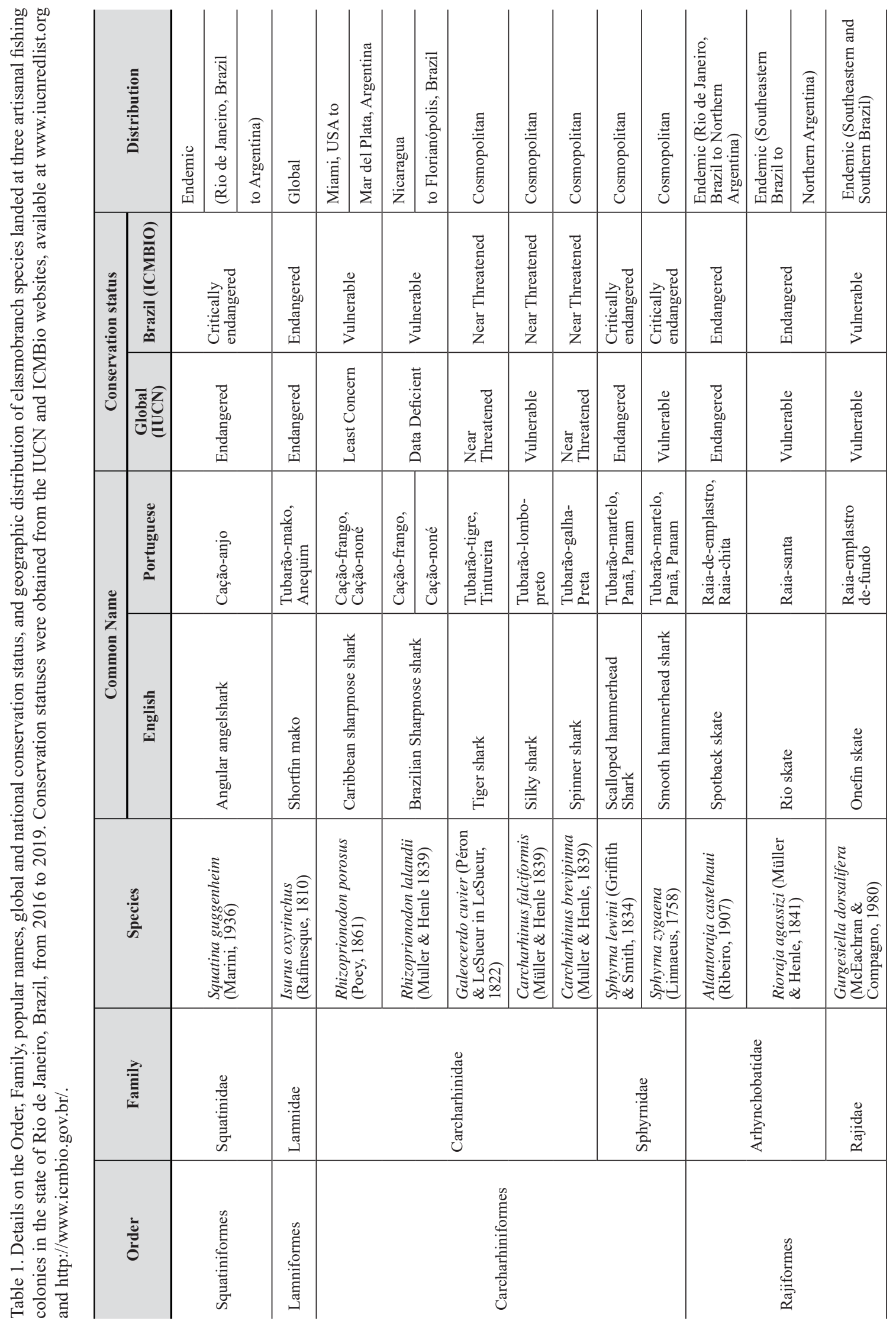




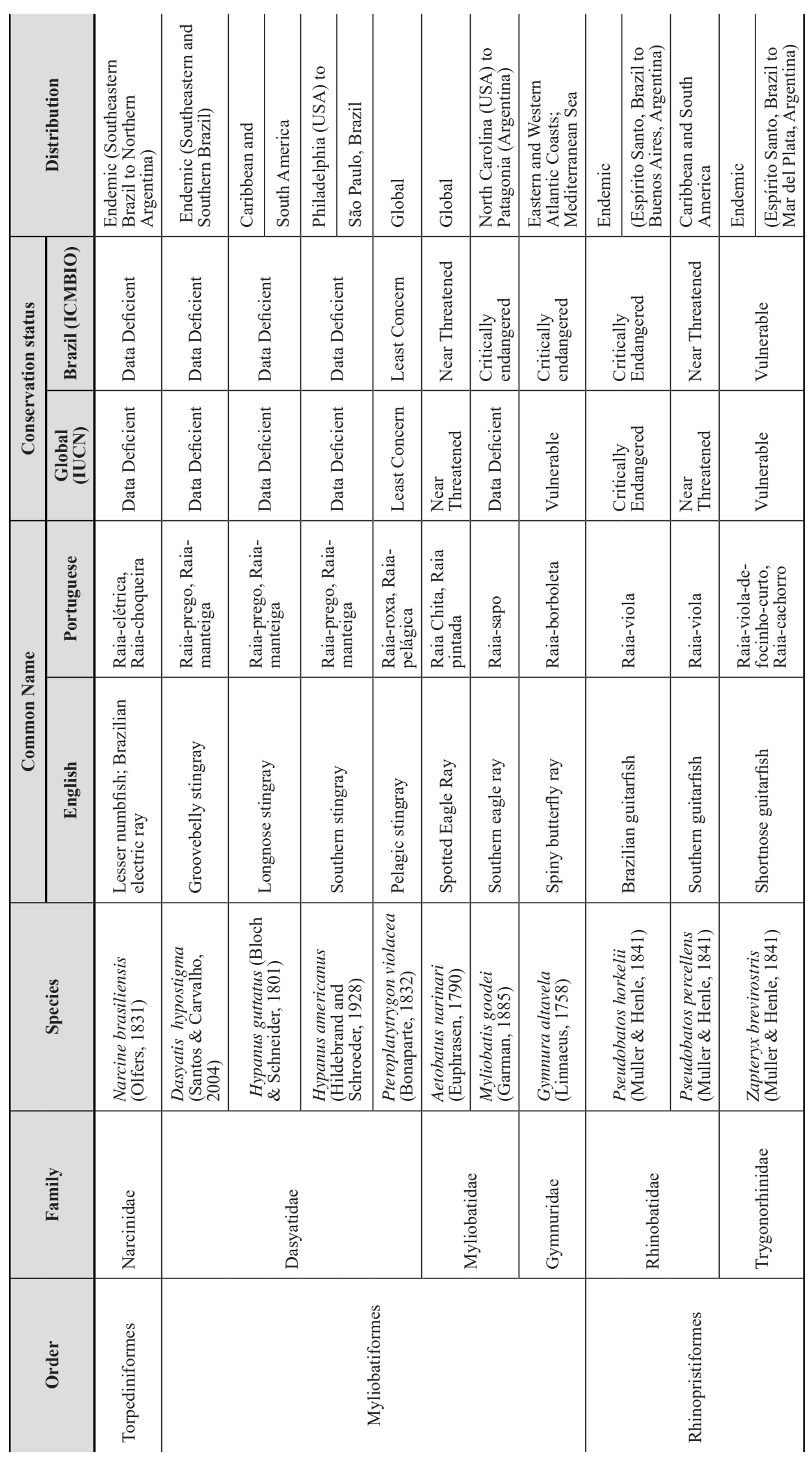


Table 2. Elasmobranch individuals landed at three artisanal fishing colonies in the state of Rio de Janeiro, Brazil, from 2016 to 2019.

\begin{tabular}{|c|c|c|c|c|}
\hline Species & Sample Code & Sample Site & $\begin{array}{l}\text { Collection } \\
\text { Day }\end{array}$ & Source \\
\hline Squatina guggenheim & $\mathrm{SC} 013 \dagger$ & Cais de Tamoios, Cabo Frio & - & - \\
\hline \multirow[t]{2}{*}{ (Marini, 1936) } & HDRG045† & $\begin{array}{c}\text { Colônia de Pesca Z-13, Posto 6, } \\
\text { Copacabana }\end{array}$ & $20 / 12 / 18$ & Sample \\
\hline & VB05* & Colônia de Pesca Z-7, Itaipu & $06 / 03 / 16$ & Photo \\
\hline Isurus oxyrinchus & HDRG008† & $\begin{array}{c}\text { Colônia de Pesca Z-13, Posto 6, } \\
\text { Copacabana }\end{array}$ & $30 / 09 / 18$ & Sample \\
\hline (Rafinesque, 1810) & VB19* & Colônia de Pesca Z-7, Itaipu & $07 / 08 / 16$ & Photo \\
\hline Rhizoprionodon porosus & DZ-UERJ ${ }^{\#}$ & Cais de Tamoios, Cabo Frio & $08 / 11 / 17$ & Sample \\
\hline \multirow[t]{28}{*}{ (Poey, 1861) } & DZ-UERJ ${ }^{\#}$ & Cais de Tamoios, Cabo Frio & $14 / 12 / 17$ & Sample \\
\hline & DZ-UERJ ${ }^{\#}$ & Cais de Tamoios, Cabo Frio & $14 / 12 / 17$ & Sample \\
\hline & DZ-UERJ ${ }^{\#}$ & Cais de Tamoios, Cabo Frio & $14 / 12 / 17$ & Sample \\
\hline & DZ-UERJ ${ }^{\#}$ & Cais de Tamoios, Cabo Frio & $14 / 12 / 17$ & Sample \\
\hline & DZ-UERJ ${ }^{\#}$ & Cais de Tamoios, Cabo Frio & $14 / 12 / 17$ & Sample \\
\hline & DZ-UERJ ${ }^{\#}$ & Cais de Tamoios, Cabo Frio & $14 / 12 / 17$ & Sample \\
\hline & DZ-UERJ ${ }^{\#}$ & Cais de Tamoios, Cabo Frio & $14 / 12 / 17$ & Sample \\
\hline & DZ-UERJ ${ }^{\#}$ & Cais de Tamoios, Cabo Frio & $01 / 02 / 18$ & Sample \\
\hline & DZ-UERJ ${ }^{\#}$ & Cais de Tamoios, Cabo Frio & $01 / 02 / 18$ & Sample \\
\hline & DZ-UERJ ${ }^{\#}$ & Cais de Tamoios, Cabo Frio & $01 / 02 / 18$ & Sample \\
\hline & DZ-UERJ ${ }^{\#}$ & Cais de Tamoios, Cabo Frio & $01 / 02 / 18$ & Sample \\
\hline & DZ-UERJ ${ }^{\#}$ & Cais de Tamoios, Cabo Frio & $01 / 02 / 18$ & Sample \\
\hline & DZ-UERJ ${ }^{\#}$ & Cais de Tamoios, Cabo Frio & $18 / 01 / 18$ & Sample \\
\hline & DZ-UERJ ${ }^{\#}$ & Cais de Tamoios, Cabo Frio & $18 / 01 / 18$ & Sample \\
\hline & DZ-UERJ ${ }^{\#}$ & Cais de Tamoios, Cabo Frio & $18 / 01 / 18$ & Sample \\
\hline & DZ-UERJ ${ }^{\#}$ & Cais de Tamoios, Cabo Frio & $18 / 01 / 18$ & Sample \\
\hline & DZ-UERJ ${ }^{\#}$ & Cais de Tamoios, Cabo Frio & $18 / 01 / 18$ & Sample \\
\hline & DZ-UERJ ${ }^{\#}$ & Cais de Tamoios, Cabo Frio & $18 / 01 / 18$ & Sample \\
\hline & DZ-UERJ ${ }^{\#}$ & Cais de Tamoios, Cabo Frio & $18 / 01 / 18$ & Sample \\
\hline & DZ-UERJ ${ }^{\#}$ & Cais de Tamoios, Cabo Frio & $15 / 06 / 18$ & Sample \\
\hline & DZ-UERJ ${ }^{\#}$ & Cais de Tamoios, Cabo Frio & $15 / 06 / 18$ & Sample \\
\hline & DZ-UERJ ${ }^{\#}$ & Cais de Tamoios, Cabo Frio & $15 / 06 / 18$ & Sample \\
\hline & DZ-UERJ ${ }^{\#}$ & Cais de Tamoios, Cabo Frio & $13 / 07 / 18$ & Sample \\
\hline & DZ-UERJ ${ }^{\#}$ & Cais de Tamoios, Cabo Frio & $13 / 07 / 18$ & Sample \\
\hline & DZ-UERJ ${ }^{\#}$ & Cais de Tamoios, Cabo Frio & $13 / 07 / 18$ & Sample \\
\hline & DZ-UERJ $^{\#}$ & Cais de Tamoios, Cabo Frio & $13 / 07 / 18$ & Sample \\
\hline & DZ-UERJ ${ }^{\#}$ & Cais de Tamoios, Cabo Frio & $13 / 07 / 18$ & Sample \\
\hline & DZ-UERJ ${ }^{\#}$ & Cais de Tamoios, Cabo Frio & $13 / 07 / 18$ & Sample \\
\hline
\end{tabular}




\begin{tabular}{|c|c|c|c|c|}
\hline Species & Sample Code & Sample Site & $\begin{array}{l}\text { Collection } \\
\text { Day }\end{array}$ & Source \\
\hline & DZ-UERJ ${ }^{\#}$ & Cais de Tamoios, Cabo Frio & $16 / 08 / 18$ & Sample \\
\hline & DZ-UERJ ${ }^{\#}$ & Cais de Tamoios, Cabo Frio & $16 / 08 / 18$ & Sample \\
\hline & DZ-UERJ ${ }^{\#}$ & Cais de Tamoios, Cabo Frio & $16 / 08 / 18$ & Sample \\
\hline & DZ-UERJ ${ }^{\#}$ & Cais de Tamoios, Cabo Frio & $16 / 08 / 18$ & Sample \\
\hline & DZ-UERJ ${ }^{\#}$ & Cais de Tamoios, Cabo Frio & $16 / 08 / 18$ & Sample \\
\hline & DZ-UERJ ${ }^{\#}$ & Cais de Tamoios, Cabo Frio & $16 / 08 / 18$ & Sample \\
\hline & DZ-UERJ ${ }^{\#}$ & Cais de Tamoios, Cabo Frio & $16 / 08 / 18$ & Sample \\
\hline & DZ-UERJ ${ }^{\#}$ & Cais de Tamoios, Cabo Frio & $16 / 08 / 18$ & Sample \\
\hline & DZ-UERJ ${ }^{\#}$ & Cais de Tamoios, Cabo Frio & $16 / 08 / 18$ & Sample \\
\hline & DZ-UERJ ${ }^{\#}$ & Cais de Tamoios, Cabo Frio & $16 / 08 / 18$ & Sample \\
\hline & DZ-UERJ ${ }^{\#}$ & Cais de Tamoios, Cabo Frio & $16 / 08 / 18$ & Sample \\
\hline & DZ-UERJ ${ }^{\#}$ & Cais de Tamoios, Cabo Frio & $16 / 08 / 18$ & Sample \\
\hline & DZ-UERJ ${ }^{\#}$ & Cais de Tamoios, Cabo Frio & $16 / 08 / 18$ & Sample \\
\hline & DZ-UERJ ${ }^{\#}$ & Cais de Tamoios, Cabo Frio & $16 / 08 / 18$ & Sample \\
\hline & $\mathrm{HDRG} 062 \uparrow$ & Cais de Tamoios, Cabo Frio & $19 / 01 / 19$ & Sample \\
\hline & HDRG063† & Cais de Tamoios, Cabo Frio & $19 / 01 / 19$ & Sample \\
\hline & HDRG064† & Cais de Tamoios, Cabo Frio & $19 / 01 / 19$ & Sample \\
\hline & HDRG066† & Cais de Tamoios, Cabo Frio & $19 / 01 / 19$ & Sample \\
\hline & HDRG067† & Cais de Tamoios, Cabo Frio & 19/01/19 & Sample \\
\hline & HDRG069† & Cais de Tamoios, Cabo Frio & $19 / 01 / 19$ & Sample \\
\hline & HDRG070† & Cais de Tamoios, Cabo Frio & $19 / 01 / 19$ & Sample \\
\hline & HDRG071† & Cais de Tamoios, Cabo Frio & $19 / 01 / 19$ & Sample \\
\hline & HDRG084† & Cais de Tamoios, Cabo Frio & 07/02/19 & Sample \\
\hline & HDRG085† & Cais de Tamoios, Cabo Frio & 07/02/19 & Sample \\
\hline & HDRG086† & Cais de Tamoios, Cabo Frio & $07 / 02 / 19$ & Sample \\
\hline & HDRG087† & Cais de Tamoios, Cabo Frio & 07/02/19 & Sample \\
\hline & $\mathrm{HDRG} 088 \dagger$ & Cais de Tamoios, Cabo Frio & 07/02/19 & Sample \\
\hline & HDRG089† & Cais de Tamoios, Cabo Frio & 07/02/19 & Sample \\
\hline & HDRG090† & Cais de Tamoios, Cabo Frio & $07 / 02 / 19$ & Sample \\
\hline & HDRG091† & Cais de Tamoios, Cabo Frio & 07/02/19 & Sample \\
\hline & HDRG092† & Cais de Tamoios, Cabo Frio & $07 / 02 / 19$ & Sample \\
\hline & HDRG093† & Cais de Tamoios, Cabo Frio & $07 / 02 / 19$ & Sample \\
\hline & HDRG094† & Cais de Tamoios, Cabo Frio & $07 / 02 / 19$ & Sample \\
\hline & HDRG095† & Cais de Tamoios, Cabo Frio & 07/02/19 & Sample \\
\hline & HDRG180† & Cais de Tamoios, Cabo Frio & $29 / 06 / 19$ & Sample \\
\hline \multirow{5}{*}{$\begin{array}{l}\text { Rhizoprionodon lalandii } \\
\text { (Muller \& Henle 1839) }\end{array}$} & DZ-UERJ ${ }^{\#}$ & Cais de Tamoios, Cabo Frio & $08 / 11 / 17$ & Sample \\
\hline & DZ-UERJ ${ }^{\#}$ & Cais de Tamoios, Cabo Frio & $08 / 11 / 17$ & Sample \\
\hline & DZ-UERJ ${ }^{\#}$ & Cais de Tamoios, Cabo Frio & $14 / 12 / 17$ & Sample \\
\hline & DZ-UERJ ${ }^{\#}$ & Cais de Tamoios, Cabo Frio & $14 / 12 / 17$ & Sample \\
\hline & DZ-UERJ" & Cais de Tamoios, Cabo Frio & $14 / 12 / 17$ & Sample \\
\hline
\end{tabular}




\begin{tabular}{|c|c|c|c|c|}
\hline Species & Sample Code & Sample Site & $\begin{array}{l}\text { Collection } \\
\text { Day }\end{array}$ & Source \\
\hline & DZ-UERJ ${ }^{\#}$ & Cais de Tamoios, Cabo Frio & $14 / 12 / 17$ & Sample \\
\hline & DZ-UERJ ${ }^{\#}$ & Cais de Tamoios, Cabo Frio & $01 / 02 / 18$ & Sample \\
\hline & DZ-UERJ ${ }^{\#}$ & Cais de Tamoios, Cabo Frio & $01 / 02 / 18$ & Sample \\
\hline & DZ-UERJ ${ }^{\#}$ & Cais de Tamoios, Cabo Frio & $01 / 02 / 18$ & Sample \\
\hline & DZ-UERJ ${ }^{\#}$ & Cais de Tamoios, Cabo Frio & $01 / 02 / 18$ & Sample \\
\hline & DZ-UERJ ${ }^{\#}$ & Cais de Tamoios, Cabo Frio & $01 / 02 / 18$ & Sample \\
\hline & DZ-UERJ ${ }^{\#}$ & Cais de Tamoios, Cabo Frio & $01 / 02 / 18$ & Sample \\
\hline & DZ-UERJ ${ }^{\#}$ & Cais de Tamoios, Cabo Frio & $01 / 02 / 18$ & Sample \\
\hline & DZ-UERJ ${ }^{\#}$ & Cais de Tamoios, Cabo Frio & $13 / 07 / 18$ & Sample \\
\hline & DZ-UERJ ${ }^{\#}$ & Cais de Tamoios, Cabo Frio & $13 / 07 / 18$ & Sample \\
\hline & DZ-UERJ ${ }^{\#}$ & Cais de Tamoios, Cabo Frio & $16 / 08 / 18$ & Sample \\
\hline & DZ-UERJ ${ }^{\#}$ & Cais de Tamoios, Cabo Frio & $16 / 08 / 18$ & Sample \\
\hline & DZ-UERJ ${ }^{\#}$ & Cais de Tamoios, Cabo Frio & $16 / 08 / 18$ & Sample \\
\hline & DZ-UERJ ${ }^{\#}$ & Cais de Tamoios, Cabo Frio & $16 / 08 / 18$ & Sample \\
\hline & DZ-UERJ ${ }^{\#}$ & Cais de Tamoios, Cabo Frio & $16 / 08 / 18$ & Sample \\
\hline & HDRG005* & $\begin{array}{c}\text { Colônia de Pesca Z-13, Posto 6, } \\
\text { Copacabana }\end{array}$ & $30 / 09 / 18$ & Photo \\
\hline & HDRG006* & $\begin{array}{c}\text { Colônia de Pesca Z-13, Posto 6, } \\
\text { Copacabana }\end{array}$ & $30 / 09 / 18$ & Photo \\
\hline & HDRG007* & $\begin{array}{c}\text { Colônia de Pesca Z-13, Posto 6, } \\
\text { Copacabana }\end{array}$ & $30 / 09 / 18$ & Photo \\
\hline & HDRG011* & $\begin{array}{c}\text { Colônia de Pesca Z-13, Posto 6, } \\
\text { Copacabana }\end{array}$ & $09 / 10 / 18$ & Photo \\
\hline & HDRG035† & $\begin{array}{c}\text { Colônia de Pesca Z-13, Posto 6, } \\
\text { Copacabana }\end{array}$ & $06 / 12 / 18$ & Sample \\
\hline & HDRG036† & $\begin{array}{c}\text { Colônia de Pesca Z-13, Posto 6, } \\
\text { Copacabana }\end{array}$ & $06 / 12 / 18$ & Sample \\
\hline & HDRG037† & $\begin{array}{c}\text { Colônia de Pesca Z-13, Posto 6, } \\
\text { Copacabana }\end{array}$ & $06 / 12 / 18$ & Sample \\
\hline & HDRG038† & $\begin{array}{c}\text { Colônia de Pesca Z-13, Posto 6, } \\
\text { Copacabana }\end{array}$ & $06 / 12 / 18$ & Sample \\
\hline & HDRG039† & $\begin{array}{c}\text { Colônia de Pesca Z-13, Posto 6, } \\
\text { Copacabana }\end{array}$ & $06 / 12 / 18$ & Sample \\
\hline & HDRG046† & $\begin{array}{c}\text { Colônia de Pesca Z-13, Posto 6, } \\
\text { Copacabana }\end{array}$ & $20 / 12 / 18$ & Sample \\
\hline & HDRG065† & Cais de Tamoios, Cabo Frio & $19 / 01 / 19$ & Sample \\
\hline & HDRG068† & Cais de Tamoios, Cabo Frio & $19 / 01 / 19$ & Sample \\
\hline & HDRG165† & $\begin{array}{c}\text { Colônia de Pesca Z-13, Posto 6, } \\
\text { Copacabana }\end{array}$ & $22 / 06 / 19$ & Sample \\
\hline & HDRG166† & $\begin{array}{c}\text { Colônia de Pesca Z-13, Posto 6, } \\
\text { Copacabana }\end{array}$ & $22 / 06 / 19$ & Sample \\
\hline & HDRG167† & $\begin{array}{c}\text { Colônia de Pesca Z-13, Posto 6, } \\
\text { Copacabana }\end{array}$ & $22 / 06 / 19$ & Sample \\
\hline
\end{tabular}




\begin{tabular}{|c|c|c|c|c|}
\hline Species & Sample Code & Sample Site & $\begin{array}{l}\text { Collection } \\
\text { Day }\end{array}$ & Source \\
\hline & HDRG168† & $\begin{array}{c}\text { Colônia de Pesca Z-13, Posto 6, } \\
\text { Copacabana }\end{array}$ & $22 / 06 / 19$ & Sample \\
\hline & HDRG169† & $\begin{array}{c}\text { Colônia de Pesca Z-13, Posto 6, } \\
\text { Copacabana }\end{array}$ & $25 / 06 / 19$ & Sample \\
\hline & HDRG170† & $\begin{array}{c}\text { Colônia de Pesca Z-13, Posto 6, } \\
\text { Copacabana }\end{array}$ & $25 / 06 / 19$ & Sample \\
\hline & HDRG171† & $\begin{array}{c}\text { Colônia de Pesca Z-13, Posto 6, } \\
\text { Copacabana }\end{array}$ & $25 / 06 / 19$ & Sample \\
\hline & HDRG172† & $\begin{array}{c}\text { Colônia de Pesca Z-13, Posto 6, } \\
\text { Copacabana }\end{array}$ & $25 / 06 / 19$ & Sample \\
\hline & HDRG173† & $\begin{array}{c}\text { Colônia de Pesca Z-13, Posto 6, } \\
\text { Copacabana }\end{array}$ & $25 / 06 / 19$ & Sample \\
\hline & HDRG216† & $\begin{array}{c}\text { Colônia de Pesca Z-13, Posto 6, } \\
\text { Copacabana }\end{array}$ & $12 / 09 / 19$ & Sample \\
\hline & HDRG217† & $\begin{array}{c}\text { Colônia de Pesca Z-13, Posto 6, } \\
\text { Copacabana }\end{array}$ & $12 / 09 / 19$ & Sample \\
\hline & HDRG218† & $\begin{array}{c}\text { Colônia de Pesca Z-13, Posto 6, } \\
\text { Copacabana }\end{array}$ & $12 / 09 / 19$ & Sample \\
\hline Galeocerdo cuvier & HDRG251† & $\begin{array}{c}\text { Colônia de Pesca Z-13, Posto 6, } \\
\text { Copacabana }\end{array}$ & $05 / 04 / 18$ & Sample \\
\hline $\begin{array}{l}\text { (Péron \& LeSueur in } \\
\text { LeSueur, 1822) }\end{array}$ & HDRG250† & $\begin{array}{c}\text { Colônia de Pesca Z-13, Posto 6, } \\
\text { Copacabana }\end{array}$ & $20 / 09 / 19$ & Sample \\
\hline $\begin{array}{c}\text { Carcharhinus } \\
\text { falciformis } \\
\text { (Müller \& Henle 1839) }\end{array}$ & VB17* & Colônia de Pesca Z-7, Itaipu & $12 / 07 / 16$ & Photo \\
\hline Carcharhinus brevipinna & HDRG072† & $\begin{array}{c}\text { Colônia de Pesca Z-13, Posto 6, } \\
\text { Copacabana }\end{array}$ & $21 / 01 / 19$ & Sample \\
\hline \multirow[t]{9}{*}{ (Muller \& Henle, 1839) } & HDRG073† & $\begin{array}{c}\text { Colônia de Pesca Z-13, Posto 6, } \\
\text { Copacabana }\end{array}$ & $22 / 01 / 19$ & Sample \\
\hline & HDRG074† & $\begin{array}{c}\text { Colônia de Pesca Z-13, Posto 6, } \\
\text { Copacabana }\end{array}$ & $23 / 01 / 19$ & Sample \\
\hline & HDRG127† & $\begin{array}{c}\text { Colônia de Pesca Z-13, Posto 6, } \\
\text { Copacabana }\end{array}$ & $11 / 04 / 19$ & Sample \\
\hline & HDRG140† & $\begin{array}{c}\text { Colônia de Pesca Z-13, Posto 6, } \\
\text { Copacabana }\end{array}$ & $16 / 04 / 19$ & Sample \\
\hline & HDRG190* & $\begin{array}{c}\text { Colônia de Pesca Z-13, Posto 6, } \\
\text { Copacabana }\end{array}$ & 26/07/19 & Photo \\
\hline & HDRG191* & $\begin{array}{c}\text { Colônia de Pesca Z-13, Posto 6, } \\
\text { Copacabana }\end{array}$ & 26/07/19 & Photo \\
\hline & VB06* & Colônia de Pesca Z-7, Itaipu & $11 / 05 / 16$ & Photo \\
\hline & VB12* & Colônia de Pesca Z-7, Itaipu & 01/06/16 & Photo \\
\hline & VB18* & Colônia de Pesca Z-7, Itaipu & $20 / 07 / 16$ & Photo \\
\hline \multirow{3}{*}{ (Griffith \& Smith, 1834) } & $\mathrm{SC} 006 \dagger$ & Cais de Tamoios, Cabo Frio & $16 / 01 / 18$ & Sample \\
\hline & HDRG010† & $\begin{array}{c}\text { Colônia de Pesca Z-13, Posto 6, } \\
\text { Copacabana }\end{array}$ & $04 / 10 / 18$ & Sample \\
\hline & HDRG013† & $\begin{array}{c}\text { Colônia de Pesca Z-13, Posto 6, } \\
\text { Copacabana }\end{array}$ & $13 / 10 / 18$ & Sample \\
\hline
\end{tabular}




\begin{tabular}{|c|c|c|c|c|}
\hline Species & Sample Code & Sample Site & $\begin{array}{l}\text { Collection } \\
\text { Day }\end{array}$ & Source \\
\hline & HDRG034 $\uparrow$ & $\begin{array}{c}\text { Colônia de Pesca Z-13, Posto 6, } \\
\text { Copacabana }\end{array}$ & $06 / 12 / 18$ & Sample \\
\hline & HDRG183* & $\begin{array}{c}\text { Colônia de Pesca Z-13, Posto 6, } \\
\text { Copacabana }\end{array}$ & 08/07/19 & Photo \\
\hline & HDRG184* & $\begin{array}{c}\text { Colônia de Pesca Z-13, Posto 6, } \\
\text { Copacabana }\end{array}$ & 08/07/19 & Photo \\
\hline & HDRG185* & $\begin{array}{c}\text { Colônia de Pesca Z-13, Posto 6, } \\
\text { Copacabana }\end{array}$ & $11 / 07 / 19$ & Photo \\
\hline & HDRG189* & $\begin{array}{c}\text { Colônia de Pesca Z-13, Posto 6, } \\
\text { Copacabana }\end{array}$ & $26 / 07 / 19$ & Photo \\
\hline & HDRG $220 \dagger$ & $\begin{array}{c}\text { Colônia de Pesca Z-13, Posto 6, } \\
\text { Copacabana }\end{array}$ & $14 / 09 / 19$ & Sample \\
\hline & VB07* & Colônia de Pesca Z-7, Itaipu & $21 / 05 / 16$ & Photo \\
\hline & VB08* & Colônia de Pesca Z-7, Itaipu & $21 / 05 / 16$ & Photo \\
\hline & VB09* & Colônia de Pesca Z-7, Itaipu & $21 / 05 / 16$ & Photo \\
\hline & VB11* & Colônia de Pesca Z-7, Itaipu & $21 / 05 / 16$ & Photo \\
\hline & VB13* & Colônia de Pesca Z-7, Itaipu & $25 / 06 / 16$ & Photo \\
\hline & VB16* & Colônia de Pesca Z-7, Itaipu & $12 / 07 / 16$ & Photo \\
\hline & VB03* & Colônia de Pesca Z-7, Itaipu & Sem data & Photo \\
\hline Sphyrna zygaena & HDRG192† & $\begin{array}{c}\text { Colônia de Pesca Z-13, Posto 6, } \\
\text { Copacabana }\end{array}$ & $16 / 08 / 19$ & Sample \\
\hline \multirow[t]{4}{*}{ (Linnaeus, 1758) } & HDRG219† & $\begin{array}{c}\text { Colônia de Pesca Z-13, Posto 6, } \\
\text { Copacabana }\end{array}$ & $14 / 09 / 19$ & Sample \\
\hline & VB15* & Colônia de Pesca Z-7, Itaipu & $12 / 07 / 16$ & Photo \\
\hline & VB01* & Colônia de Pesca Z-7, Itaipu & Sem data & Photo \\
\hline & VB02* & Colônia de Pesca Z-7, Itaipu & Sem data & Photo \\
\hline Atlantoraja castelnaui & $\mathrm{SC} 007 \dagger$ & Cais de Tamoios, Cabo Frio & $18 / 01 / 18$ & Sample \\
\hline \multirow[t]{9}{*}{ (Ribeiro, 1907) } & $\mathrm{SC} 008 \dagger$ & Cais de Tamoios, Cabo Frio & $16 / 01 / 18$ & Sample \\
\hline & HDRG009* & $\begin{array}{c}\text { Colônia de Pesca Z-13, Posto 6, } \\
\text { Copacabana }\end{array}$ & $02 / 10 / 18$ & Photo \\
\hline & HDRG012* & $\begin{array}{c}\text { Colônia de Pesca Z-13, Posto 6, } \\
\text { Copacabana }\end{array}$ & $11 / 10 / 18$ & Photo \\
\hline & HDRG040† & $\begin{array}{c}\text { Colônia de Pesca Z-13, Posto 6, } \\
\text { Copacabana }\end{array}$ & $13 / 12 / 18$ & Sample \\
\hline & HDRG041† & $\begin{array}{c}\text { Colônia de Pesca Z-13, Posto 6, } \\
\text { Copacabana }\end{array}$ & $13 / 12 / 18$ & Sample \\
\hline & HDRG048† & $\begin{array}{c}\text { Colônia de Pesca Z-13, Posto 6, } \\
\text { Copacabana }\end{array}$ & 08/01/19 & Sample \\
\hline & HDRG200* & $\begin{array}{c}\text { Colônia de Pesca Z-13, Posto 6, } \\
\text { Copacabana }\end{array}$ & 08/09/19 & Photo \\
\hline & HDRG232* & $\begin{array}{c}\text { Colônia de Pesca Z-13, Posto 6, } \\
\text { Copacabana }\end{array}$ & $17 / 09 / 19$ & Photo \\
\hline & HDRG233* & $\begin{array}{c}\text { Colônia de Pesca Z-13, Posto 6, } \\
\text { Copacabana }\end{array}$ & $17 / 09 / 19$ & Photo \\
\hline
\end{tabular}




\begin{tabular}{|c|c|c|c|c|}
\hline Species & Sample Code & Sample Site & $\begin{array}{l}\text { Collection } \\
\text { Day }\end{array}$ & Source \\
\hline & HDRG234* & $\begin{array}{c}\text { Colônia de Pesca Z-13, Posto 6, } \\
\text { Copacabana }\end{array}$ & $17 / 09 / 19$ & Photo \\
\hline & HDRG235* & $\begin{array}{c}\text { Colônia de Pesca Z-13, Posto 6, } \\
\text { Copacabana }\end{array}$ & $17 / 09 / 19$ & Photo \\
\hline & HDRG236* & $\begin{array}{c}\text { Colônia de Pesca Z-13, Posto 6, } \\
\text { Copacabana }\end{array}$ & $17 / 09 / 19$ & Photo \\
\hline & HDRG237* & $\begin{array}{c}\text { Colônia de Pesca Z-13, Posto 6, } \\
\text { Copacabana }\end{array}$ & $17 / 09 / 19$ & Photo \\
\hline & HDRG238* & $\begin{array}{c}\text { Colônia de Pesca Z-13, Posto 6, } \\
\text { Copacabana }\end{array}$ & $17 / 09 / 19$ & Photo \\
\hline & VB14* & Colônia de Pesca Z-7, Itaipu & 06/07/16 & Photo \\
\hline \multirow{4}{*}{$\begin{array}{c}\text { Rioraja agassizi } \\
\text { (Müller \& Henle, 1841) }\end{array}$} & $\mathrm{SC} 009 \dagger$ & Cais de Tamoios, Cabo Frio & $16 / 01 / 18$ & Sample \\
\hline & $\mathrm{SC} 010 \dagger$ & Cais de Tamoios, Cabo Frio & $16 / 01 / 18$ & Sample \\
\hline & $\mathrm{SC} 011 \dagger$ & Cais de Tamoios, Cabo Frio & $16 / 01 / 18$ & Sample \\
\hline & HDRG077† & $\begin{array}{c}\text { Colônia de Pesca Z-13, Posto 6, } \\
\text { Copacabana }\end{array}$ & $23 / 01 / 19$ & Sample \\
\hline $\begin{array}{c}\text { Gurgesiella dorsalifera } \\
\text { (McEachran \& } \\
\text { Compagno, 1980) }\end{array}$ & $\mathrm{SC} 014 \uparrow$ & Cais de Tamoios, Cabo Frio & $16 / 01 / 18$ & Sample \\
\hline $\begin{array}{c}\text { Narcine brasiliensis } \\
\text { (Olfers, 1831) }\end{array}$ & $\mathrm{HDRG} 221 \dagger$ & $\begin{array}{c}\text { Colônia de Pesca Z-13, Posto 6, } \\
\text { Copacabana }\end{array}$ & $15 / 09 / 19$ & Sample \\
\hline Dasyatis hypostigma & HDRG001* & $\begin{array}{c}\text { Colônia de Pesca Z-13, Posto 6, } \\
\text { Copacabana }\end{array}$ & $22 / 09 / 18$ & Photo \\
\hline \multirow[t]{12}{*}{$\begin{array}{c}\text { (Santos \& Carvalho, } \\
\text { 2004) }\end{array}$} & HDRG019* & $\begin{array}{c}\text { Colônia de Pesca Z-13, Posto 6, } \\
\text { Copacabana }\end{array}$ & $03 / 11 / 18$ & Photo \\
\hline & HDRG020* & $\begin{array}{c}\text { Colônia de Pesca Z-13, Posto 6, } \\
\text { Copacabana }\end{array}$ & $03 / 11 / 18$ & Photo \\
\hline & HDRG021* & $\begin{array}{c}\text { Colônia de Pesca Z-13, Posto 6, } \\
\text { Copacabana }\end{array}$ & $03 / 11 / 18$ & Photo \\
\hline & HDRG022* & $\begin{array}{c}\text { Colônia de Pesca Z-13, Posto 6, } \\
\text { Copacabana }\end{array}$ & $03 / 11 / 18$ & Photo \\
\hline & HDRG023* & $\begin{array}{c}\text { Colônia de Pesca Z-13, Posto 6, } \\
\text { Copacabana }\end{array}$ & $03 / 11 / 18$ & Photo \\
\hline & HDRG024* & $\begin{array}{c}\text { Colônia de Pesca Z-13, Posto 6, } \\
\text { Copacabana }\end{array}$ & $03 / 11 / 18$ & Photo \\
\hline & HDRG025* & $\begin{array}{c}\text { Colônia de Pesca Z-13, Posto 6, } \\
\text { Copacabana }\end{array}$ & $03 / 11 / 18$ & Photo \\
\hline & HDRG026* & $\begin{array}{c}\text { Colônia de Pesca Z-13, Posto 6, } \\
\text { Copacabana }\end{array}$ & $03 / 11 / 18$ & Photo \\
\hline & HDRG027* & $\begin{array}{c}\text { Colônia de Pesca Z-13, Posto 6, } \\
\text { Copacabana }\end{array}$ & $10 / 11 / 18$ & Photo \\
\hline & HDRG028* & $\begin{array}{c}\text { Colônia de Pesca Z-13, Posto 6, } \\
\text { Copacabana }\end{array}$ & $10 / 11 / 18$ & Photo \\
\hline & HDRG029* & $\begin{array}{c}\text { Colônia de Pesca Z-13, Posto 6, } \\
\text { Copacabana }\end{array}$ & $18 / 11 / 18$ & Photo \\
\hline & HDRG030* & $\begin{array}{c}\text { Colônia de Pesca Z-13, Posto 6, } \\
\text { Copacabana }\end{array}$ & $18 / 11 / 18$ & Photo \\
\hline
\end{tabular}




\begin{tabular}{|c|c|c|c|c|}
\hline Species & Sample Code & Sample Site & $\begin{array}{l}\text { Collection } \\
\text { Day }\end{array}$ & Source \\
\hline & HDRG128† & $\begin{array}{c}\text { Colônia de Pesca Z-13, Posto 6, } \\
\text { Copacabana }\end{array}$ & $11 / 04 / 19$ & Sample \\
\hline & HDRG141† & Cais de Tamoios, Cabo Frio & $18 / 05 / 19$ & Sample \\
\hline & HDRG142† & Cais de Tamoios, Cabo Frio & $18 / 05 / 19$ & Sample \\
\hline & HDRG186* & $\begin{array}{c}\text { Colônia de Pesca Z-13, Posto 6, } \\
\text { Copacabana }\end{array}$ & $11 / 07 / 19$ & Photo \\
\hline & HDRG187* & $\begin{array}{c}\text { Colônia de Pesca Z-13, Posto 6, } \\
\text { Copacabana }\end{array}$ & $11 / 07 / 19$ & Photo \\
\hline & HDRG188* & $\begin{array}{c}\text { Colônia de Pesca Z-13, Posto 6, } \\
\text { Copacabana }\end{array}$ & $11 / 07 / 19$ & Photo \\
\hline & HDRG193* & $\begin{array}{c}\text { Colônia de Pesca Z-13, Posto 6, } \\
\text { Copacabana }\end{array}$ & $17 / 08 / 19$ & Photo \\
\hline & HDRG196* & $\begin{array}{c}\text { Colônia de Pesca Z-13, Posto 6, } \\
\text { Copacabana }\end{array}$ & $25 / 08 / 19$ & Photo \\
\hline & HDRG197* & $\begin{array}{c}\text { Colônia de Pesca Z-13, Posto 6, } \\
\text { Copacabana }\end{array}$ & $25 / 08 / 19$ & Photo \\
\hline & HDRG201† & $\begin{array}{c}\text { Colônia de Pesca Z-13, Posto 6, } \\
\text { Copacabana }\end{array}$ & 09/09/19 & Sample \\
\hline & HDRG205† & $\begin{array}{c}\text { Colônia de Pesca Z-13, Posto 6, } \\
\text { Copacabana }\end{array}$ & $11 / 09 / 19$ & Sample \\
\hline & HDRG206† & $\begin{array}{c}\text { Colônia de Pesca Z-13, Posto 6, } \\
\text { Copacabana }\end{array}$ & $11 / 09 / 19$ & Sample \\
\hline & HDRG $212 \uparrow$ & $\begin{array}{c}\text { Colônia de Pesca Z-13, Posto 6, } \\
\text { Copacabana }\end{array}$ & $12 / 09 / 19$ & Sample \\
\hline & HDRG213† & $\begin{array}{c}\text { Colônia de Pesca Z-13, Posto 6, } \\
\text { Copacabana }\end{array}$ & $12 / 09 / 19$ & Sample \\
\hline & HDRG214† & $\begin{array}{c}\text { Colônia de Pesca Z-13, Posto 6, } \\
\text { Copacabana }\end{array}$ & $12 / 09 / 19$ & Sample \\
\hline & HDRG215* & $\begin{array}{c}\text { Colônia de Pesca Z-13, Posto 6, } \\
\text { Copacabana }\end{array}$ & $12 / 09 / 19$ & Photo \\
\hline & HDRG239* & $\begin{array}{c}\text { Colônia de Pesca Z-13, Posto 6, } \\
\text { Copacabana }\end{array}$ & $17 / 09 / 19$ & Photo \\
\hline & HDRG240* & $\begin{array}{c}\text { Colônia de Pesca Z-13, Posto 6, } \\
\text { Copacabana }\end{array}$ & $17 / 09 / 19$ & Photo \\
\hline & HDRG241* & $\begin{array}{c}\text { Colônia de Pesca Z-13, Posto 6, } \\
\text { Copacabana }\end{array}$ & $17 / 09 / 19$ & Photo \\
\hline & HDRG242* & $\begin{array}{c}\text { Colônia de Pesca Z-13, Posto 6, } \\
\text { Copacabana }\end{array}$ & $17 / 09 / 19$ & Photo \\
\hline & HDRG243* & $\begin{array}{c}\text { Colônia de Pesca Z-13, Posto 6, } \\
\text { Copacabana }\end{array}$ & $17 / 09 / 19$ & Photo \\
\hline & HDRG246* & $\begin{array}{c}\text { Colônia de Pesca Z-13, Posto 6, } \\
\text { Copacabana }\end{array}$ & $18 / 09 / 19$ & Photo \\
\hline \multirow{3}{*}{$\begin{array}{l}\text { Hypanus guttatus } \\
\text { (Bloch \& Schneider, } \\
\text { 1801) }\end{array}$} & HDRG $202 \uparrow$ & $\begin{array}{c}\text { Colônia de Pesca Z-13, Posto 6, } \\
\text { Copacabana }\end{array}$ & $10 / 09 / 19$ & Sample \\
\hline & HDRG208* & $\begin{array}{c}\text { Colônia de Pesca Z-13, Posto 6, } \\
\text { Copacabana }\end{array}$ & $11 / 09 / 19$ & Photo \\
\hline & HDRG209* & $\begin{array}{c}\text { Colônia de Pesca Z-13, Posto 6, } \\
\text { Copacabana }\end{array}$ & $11 / 09 / 19$ & Photo \\
\hline
\end{tabular}




\begin{tabular}{|c|c|c|c|c|}
\hline Species & Sample Code & Sample Site & $\begin{array}{l}\text { Collection } \\
\text { Day }\end{array}$ & Source \\
\hline & HDRG210* & $\begin{array}{c}\text { Colônia de Pesca Z-13, Posto 6, } \\
\text { Copacabana }\end{array}$ & $11 / 09 / 19$ & Photo \\
\hline & HDRG211* & $\begin{array}{c}\text { Colônia de Pesca Z-13, Posto 6, } \\
\text { Copacabana }\end{array}$ & $12 / 09 / 19$ & Photo \\
\hline & HDRG222* & $\begin{array}{c}\text { Colônia de Pesca Z-13, Posto 6, } \\
\text { Copacabana }\end{array}$ & $17 / 09 / 19$ & Photo \\
\hline & HDRG223* & $\begin{array}{c}\text { Colônia de Pesca Z-13, Posto 6, } \\
\text { Copacabana }\end{array}$ & $17 / 09 / 19$ & Photo \\
\hline & HDRG224* & $\begin{array}{c}\text { Colônia de Pesca Z-13, Posto 6, } \\
\text { Copacabana }\end{array}$ & $17 / 09 / 19$ & Photo \\
\hline & HDRG225* & $\begin{array}{c}\text { Colônia de Pesca Z-13, Posto 6, } \\
\text { Copacabana }\end{array}$ & $17 / 09 / 19$ & Photo \\
\hline & HDRG226* & $\begin{array}{c}\text { Colônia de Pesca Z-13, Posto 6, } \\
\text { Copacabana }\end{array}$ & $17 / 09 / 19$ & Photo \\
\hline & HDRG227* & $\begin{array}{c}\text { Colônia de Pesca Z-13, Posto 6, } \\
\text { Copacabana }\end{array}$ & $17 / 09 / 19$ & Photo \\
\hline & HDRG228* & $\begin{array}{c}\text { Colônia de Pesca Z-13, Posto 6, } \\
\text { Copacabana }\end{array}$ & $17 / 09 / 19$ & Photo \\
\hline & HDRG229* & $\begin{array}{c}\text { Colônia de Pesca Z-13, Posto 6, } \\
\text { Copacabana }\end{array}$ & $17 / 09 / 19$ & Photo \\
\hline Hypanus americanus & HDRG198† & $\begin{array}{c}\text { Colônia de Pesca Z-13, Posto 6, } \\
\text { Copacabana }\end{array}$ & 08/09/19 & Sample \\
\hline \multirow[t]{7}{*}{$\begin{array}{l}\text { (Hildebrand and } \\
\text { Schroeder, 1928) }\end{array}$} & HDRG199† & $\begin{array}{c}\text { Colônia de Pesca Z-13, Posto 6, } \\
\text { Copacabana }\end{array}$ & 08/09/19 & Sample \\
\hline & HDRG203† & $\begin{array}{c}\text { Colônia de Pesca Z-13, Posto 6, } \\
\text { Copacabana }\end{array}$ & $10 / 09 / 19$ & Sample \\
\hline & HDRG204† & $\begin{array}{c}\text { Colônia de Pesca Z-13, Posto 6, } \\
\text { Copacabana }\end{array}$ & $10 / 09 / 19$ & Sample \\
\hline & HDRG230* & $\begin{array}{c}\text { Colônia de Pesca Z-13, Posto 6, } \\
\text { Copacabana }\end{array}$ & $17 / 09 / 19$ & Photo \\
\hline & HDRG231* & $\begin{array}{c}\text { Colônia de Pesca Z-13, Posto 6, } \\
\text { Copacabana }\end{array}$ & $17 / 09 / 19$ & Photo \\
\hline & HDRG244* & $\begin{array}{c}\text { Colônia de Pesca Z-13, Posto 6, } \\
\text { Copacabana }\end{array}$ & $18 / 09 / 19$ & Photo \\
\hline & HDRG245* & $\begin{array}{c}\text { Colônia de Pesca Z-13, Posto 6, } \\
\text { Copacabana }\end{array}$ & $18 / 09 / 19$ & Photo \\
\hline $\begin{array}{c}\text { Pteroplatytrygon } \\
\text { violacea } \\
\text { (Bonaparte, 1832) }\end{array}$ & HDRG123* & $\begin{array}{c}\text { Colônia de Pesca Z-13, Posto 6, } \\
\text { Copacabana }\end{array}$ & 08/04/19 & Photo \\
\hline Gymnura altavela & HDRG003* & $\begin{array}{c}\text { Colônia de Pesca Z-13, Posto 6, } \\
\text { Copacabana }\end{array}$ & $27 / 09 / 18$ & Photo \\
\hline \multirow[t]{3}{*}{ (Linnaeus, 1758) } & HDRG004* & $\begin{array}{c}\text { Colônia de Pesca Z-13, Posto 6, } \\
\text { Copacabana }\end{array}$ & $27 / 09 / 18$ & Photo \\
\hline & HDRG015* & $\begin{array}{c}\text { Colônia de Pesca Z-13, Posto 6, } \\
\text { Copacabana }\end{array}$ & $19 / 10 / 18$ & Photo \\
\hline & HDRG016* & $\begin{array}{c}\text { Colônia de Pesca Z-13, Posto 6, } \\
\text { Copacabana }\end{array}$ & $19 / 10 / 18$ & Photo \\
\hline
\end{tabular}




\begin{tabular}{|c|c|c|c|c|}
\hline Species & Sample Code & Sample Site & $\begin{array}{l}\text { Collection } \\
\text { Day }\end{array}$ & Source \\
\hline & HDRG017* & $\begin{array}{c}\text { Colônia de Pesca Z-13, Posto 6, } \\
\text { Copacabana }\end{array}$ & $19 / 10 / 18$ & Photo \\
\hline & HDRG047† & $\begin{array}{c}\text { Colônia de Pesca Z-13, Posto 6, } \\
\text { Copacabana }\end{array}$ & $20 / 12 / 18$ & Sample \\
\hline & HDRG096* & $\begin{array}{c}\text { Colônia de Pesca Z-13, Posto 6, } \\
\text { Copacabana }\end{array}$ & $02 / 04 / 19$ & Photo \\
\hline & HDRG147* & $\begin{array}{c}\text { Colônia de Pesca Z-13, Posto 6, } \\
\text { Copacabana }\end{array}$ & $31 / 05 / 19$ & Photo \\
\hline & HDRG161* & $\begin{array}{c}\text { Colônia de Pesca Z-13, Posto 6, } \\
\text { Copacabana }\end{array}$ & $12 / 06 / 19$ & Photo \\
\hline & HDRG162* & $\begin{array}{c}\text { Colônia de Pesca Z-13, Posto 6, } \\
\text { Copacabana }\end{array}$ & $12 / 06 / 19$ & Photo \\
\hline & HDRG181† & Cais de Tamoios, Cabo Frio & $29 / 06 / 19$ & Sample \\
\hline & HDRG194* & $\begin{array}{c}\text { Colônia de Pesca Z-13, Posto 6, } \\
\text { Copacabana }\end{array}$ & $25 / 08 / 19$ & Photo \\
\hline & HDRG195* & $\begin{array}{c}\text { Colônia de Pesca Z-13, Posto 6, } \\
\text { Copacabana }\end{array}$ & $25 / 08 / 19$ & Photo \\
\hline & HDRG248* & $\begin{array}{c}\text { Colônia de Pesca Z-13, Posto 6, } \\
\text { Copacabana }\end{array}$ & $19 / 09 / 19$ & Photo \\
\hline $\begin{array}{l}\text { Aetobatus narinari } \\
\text { (Euphrasen, 1790) }\end{array}$ & HDRG253* & Cais de Tamoios, Cabo Frio & $19 / 01 / 19$ & Photo \\
\hline $\begin{array}{l}\text { Myliobatis goodei } \\
\text { (Garman, 1885) }\end{array}$ & HDRG25† & Cais de Tamoios, Cabo Frio & $29 / 06 / 19$ & Sample \\
\hline Pseudobatos horkelii & $\mathrm{SC} 001 \uparrow$ & Cais de Tamoios, Cabo Frio & $18 / 01 / 18$ & Sample \\
\hline \multirow[t]{14}{*}{ (Muller \& Henle, 1841) } & $\mathrm{SC} 002 \uparrow$ & Cais de Tamoios, Cabo Frio & $18 / 01 / 18$ & Sample \\
\hline & $\mathrm{SC} 003 \dagger$ & Cais de Tamoios, Cabo Frio & $01 / 02 / 18$ & Sample \\
\hline & $\mathrm{SC} 004 \uparrow$ & Cais de Tamoios, Cabo Frio & $01 / 02 / 18$ & Sample \\
\hline & $\mathrm{SC} 005 \dagger$ & Cais de Tamoios, Cabo Frio & $01 / 02 / 18$ & Sample \\
\hline & HDRG044† & $\begin{array}{c}\text { Colônia de Pesca Z-13, Posto 6, } \\
\text { Copacabana }\end{array}$ & $13 / 12 / 18$ & Sample \\
\hline & HDRG056† & $\begin{array}{c}\text { Colônia de Pesca Z-13, Posto 6, } \\
\text { Copacabana }\end{array}$ & $17 / 01 / 19$ & Sample \\
\hline & HDRG057† & $\begin{array}{c}\text { Colônia de Pesca Z-13, Posto 6, } \\
\text { Copacabana }\end{array}$ & $17 / 01 / 19$ & Sample \\
\hline & HDRG058† & $\begin{array}{c}\text { Colônia de Pesca Z-13, Posto 6, } \\
\text { Copacabana }\end{array}$ & $17 / 01 / 19$ & Sample \\
\hline & HDRG174 $\uparrow$ & Cais de Tamoios, Cabo Frio & $29 / 06 / 19$ & Sample \\
\hline & HDRG175† & Cais de Tamoios, Cabo Frio & $29 / 06 / 19$ & Sample \\
\hline & HDRG176† & Cais de Tamoios, Cabo Frio & $29 / 06 / 19$ & Sample \\
\hline & HDRG177† & Cais de Tamoios, Cabo Frio & $29 / 06 / 19$ & Sample \\
\hline & HDRG178† & Cais de Tamoios, Cabo Frio & $29 / 06 / 19$ & Sample \\
\hline & VB10* & Colônia de Pesca Z-7, Itaipu & $21 / 05 / 16$ & Photo \\
\hline Pseudobatos percellens & HDRG014* & $\begin{array}{c}\text { Colônia de Pesca Z-13, Posto 6, } \\
\text { Copacabana }\end{array}$ & $13 / 10 / 18$ & Photo \\
\hline
\end{tabular}




\begin{tabular}{|c|c|c|c|c|}
\hline Species & Sample Code & Sample Site & $\begin{array}{l}\text { Collection } \\
\text { Day }\end{array}$ & Source \\
\hline \multirow[t]{24}{*}{ (Muller \& Henle, 1841) } & HDRG018* & $\begin{array}{c}\text { Colônia de Pesca Z-13, Posto 6, } \\
\text { Copacabana }\end{array}$ & $25 / 10 / 18$ & Photo \\
\hline & HDRG031* & $\begin{array}{c}\text { Colônia de Pesca Z-13, Posto 6, } \\
\text { Copacabana }\end{array}$ & $29 / 11 / 18$ & Photo \\
\hline & HDRG032* & $\begin{array}{c}\text { Colônia de Pesca Z-13, Posto 6, } \\
\text { Copacabana }\end{array}$ & $29 / 11 / 18$ & Photo \\
\hline & HDRG033* & $\begin{array}{c}\text { Colônia de Pesca Z-13, Posto 6, } \\
\text { Copacabana }\end{array}$ & $29 / 11 / 18$ & Photo \\
\hline & HDRG042† & $\begin{array}{c}\text { Colônia de Pesca Z-13, Posto 6, } \\
\text { Copacabana }\end{array}$ & $13 / 12 / 18$ & Sample \\
\hline & HDRG043† & $\begin{array}{c}\text { Colônia de Pesca Z-13, Posto 6, } \\
\text { Copacabana }\end{array}$ & $13 / 12 / 18$ & Sample \\
\hline & HDRG049† & $\begin{array}{c}\text { Colônia de Pesca Z-13, Posto 6, } \\
\text { Copacabana }\end{array}$ & 08/01/19 & Sample \\
\hline & HDRG050† & $\begin{array}{c}\text { Colônia de Pesca Z-13, Posto 6, } \\
\text { Copacabana }\end{array}$ & 08/01/19 & Sample \\
\hline & HDRG051† & $\begin{array}{c}\text { Colônia de Pesca Z-13, Posto 6, } \\
\text { Copacabana }\end{array}$ & $11 / 01 / 19$ & Sample \\
\hline & HDRG052† & $\begin{array}{c}\text { Colônia de Pesca Z-13, Posto 6, } \\
\text { Copacabana }\end{array}$ & $11 / 01 / 19$ & Sample \\
\hline & HDRG059† & $\begin{array}{c}\text { Colônia de Pesca Z-13, Posto 6, } \\
\text { Copacabana }\end{array}$ & $17 / 01 / 19$ & Sample \\
\hline & HDRG060† & $\begin{array}{c}\text { Colônia de Pesca Z-13, Posto 6, } \\
\text { Copacabana }\end{array}$ & $17 / 01 / 19$ & Sample \\
\hline & HDRG061† & $\begin{array}{c}\text { Colônia de Pesca Z-13, Posto 6, } \\
\text { Copacabana }\end{array}$ & $17 / 01 / 19$ & Sample \\
\hline & HDRG082* & $\begin{array}{c}\text { Colônia de Pesca Z-13, Posto 6, } \\
\text { Copacabana }\end{array}$ & $01 / 02 / 19$ & Photo \\
\hline & HDRG083* & $\begin{array}{c}\text { Colônia de Pesca Z-13, Posto 6, } \\
\text { Copacabana }\end{array}$ & $01 / 02 / 19$ & Photo \\
\hline & HDRG124* & $\begin{array}{c}\text { Colônia de Pesca Z-13, Posto 6, } \\
\text { Copacabana }\end{array}$ & $11 / 04 / 19$ & Photo \\
\hline & HDRG125* & $\begin{array}{c}\text { Colônia de Pesca Z-13, Posto 6, } \\
\text { Copacabana }\end{array}$ & $11 / 04 / 19$ & Photo \\
\hline & HDRG126* & $\begin{array}{c}\text { Colônia de Pesca Z-13, Posto 6, } \\
\text { Copacabana }\end{array}$ & $11 / 04 / 19$ & Photo \\
\hline & HDRG129* & $\begin{array}{c}\text { Colônia de Pesca Z-13, Posto 6, } \\
\text { Copacabana }\end{array}$ & $15 / 04 / 19$ & Photo \\
\hline & HDRG130* & $\begin{array}{c}\text { Colônia de Pesca Z-13, Posto 6, } \\
\text { Copacabana }\end{array}$ & $15 / 04 / 19$ & Photo \\
\hline & HDRG131* & $\begin{array}{c}\text { Colônia de Pesca Z-13, Posto 6, } \\
\text { Copacabana }\end{array}$ & $15 / 04 / 19$ & Photo \\
\hline & HDRG132* & $\begin{array}{c}\text { Colônia de Pesca Z-13, Posto 6, } \\
\text { Copacabana }\end{array}$ & $15 / 04 / 19$ & Photo \\
\hline & HDRG133* & $\begin{array}{c}\text { Colônia de Pesca Z-13, Posto 6, } \\
\text { Copacabana }\end{array}$ & $15 / 04 / 19$ & Photo \\
\hline & HDRG134* & $\begin{array}{c}\text { Colônia de Pesca Z-13, Posto 6, } \\
\text { Copacabana }\end{array}$ & $15 / 04 / 19$ & Photo \\
\hline
\end{tabular}




\begin{tabular}{|c|c|c|c|c|}
\hline Species & Sample Code & Sample Site & $\begin{array}{c}\text { Collection } \\
\text { Day }\end{array}$ & Source \\
\hline & HDRG135* & $\begin{array}{c}\text { Colônia de Pesca Z-13, Posto 6, } \\
\text { Copacabana }\end{array}$ & $15 / 04 / 19$ & Photo \\
\hline & HDRG136* & $\begin{array}{c}\text { Colônia de Pesca Z-13, Posto 6, } \\
\text { Copacabana }\end{array}$ & $15 / 04 / 19$ & Photo \\
\hline & HDRG137* & $\begin{array}{c}\text { Colônia de Pesca Z-13, Posto 6, } \\
\text { Copacabana }\end{array}$ & $15 / 04 / 19$ & Photo \\
\hline & HDRG138* & $\begin{array}{c}\text { Colônia de Pesca Z-13, Posto 6, } \\
\text { Copacabana }\end{array}$ & $15 / 04 / 19$ & Photo \\
\hline & HDRG139* & $\begin{array}{c}\text { Colônia de Pesca Z-13, Posto 6, } \\
\text { Copacabana }\end{array}$ & $15 / 04 / 19$ & Photo \\
\hline & HDRG179† & Cais de Tamoios, Cabo Frio & $29 / 06 / 19$ & Sample \\
\hline Zapteryx brevirostris & HDRG075† & $\begin{array}{c}\text { Colônia de Pesca Z-13, Posto 6, } \\
\text { Copacabana }\end{array}$ & $23 / 01 / 19$ & Sample \\
\hline \multirow[t]{4}{*}{ (Muller \& Henle, 1841) } & HDRG076† & $\begin{array}{c}\text { Colônia de Pesca Z-13, Posto 6, } \\
\text { Copacabana }\end{array}$ & $23 / 01 / 19$ & Sample \\
\hline & HDRG247* & $\begin{array}{c}\text { Colônia de Pesca Z-13, Posto 6, } \\
\text { Copacabana }\end{array}$ & $19 / 09 / 19$ & Photo \\
\hline & HDRG249† & $\begin{array}{c}\text { Colônia de Pesca Z-13, Posto 6, } \\
\text { Copacabana }\end{array}$ & $20 / 09 / 19$ & Sample \\
\hline & $\mathrm{SC} 012 \dagger$ & Cais de Tamoios, Cabo Frio & $18 / 01 / 18$ & Sample \\
\hline
\end{tabular}

* indicates a photo source; $\uparrow$ indicates a sample source.; \# UERJ Ichthyological Collection.

are presented in Table 1, while data concerning elasmobranch individuals landed at three artisanal fishing colonies in the state of Rio de Janeiro, Brazil, from 2016 to 2019 are presented in Table 2.

Frequency data for each elasmobranch species landed at the Tamoios, Z-7 and Z-13 artisanal colonies is displayed in Figure 2. It is important to note that no standardized sampling effort was carried out in this study, so no frequency proportion extrapolation in possible for the analyzed areas.

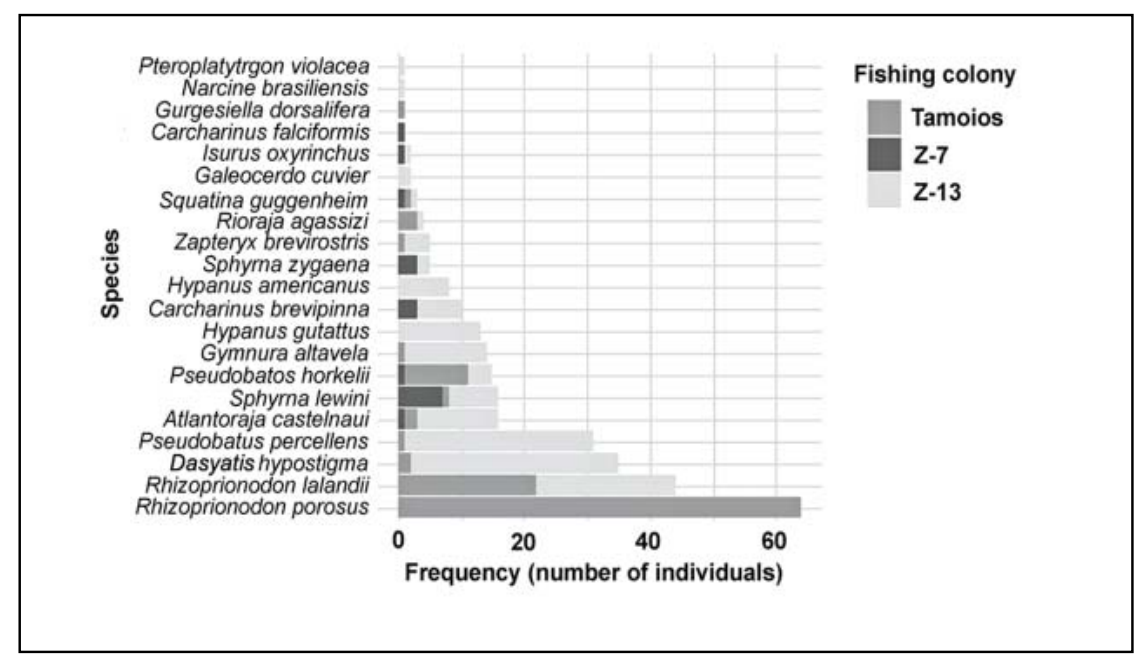

Figure 2. Frequency data for each elasmobranch species landed at the Tamoios, Z-7 and Z-13 artisanal colonies. 


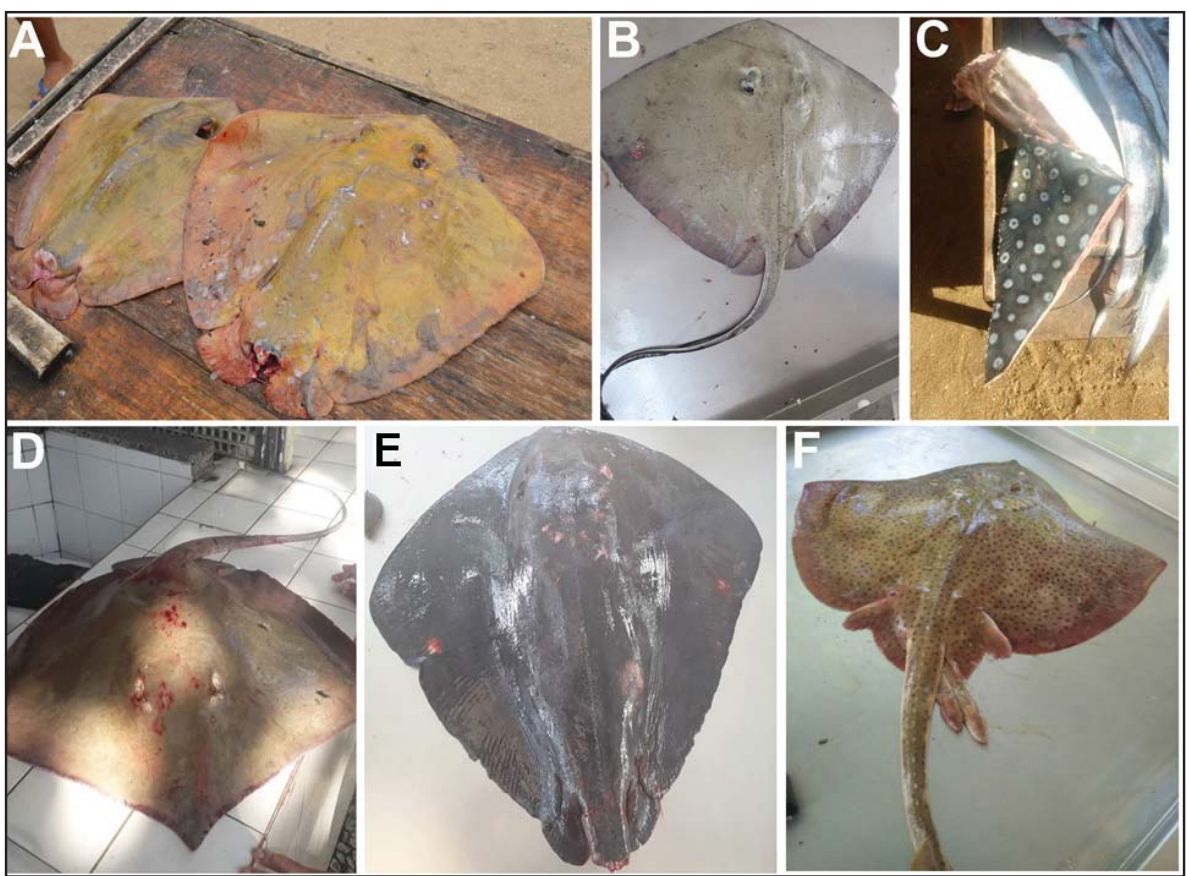

Figure 3. Ray species identified during this study. (A) Dasyatis hypostigma; (B) Hypanus americanus; (C) Aetobatus narinari; (D) Hypanus guttatus; (E) Pteroplatrygon violacea; (F) Atlantoraja castelnaui.
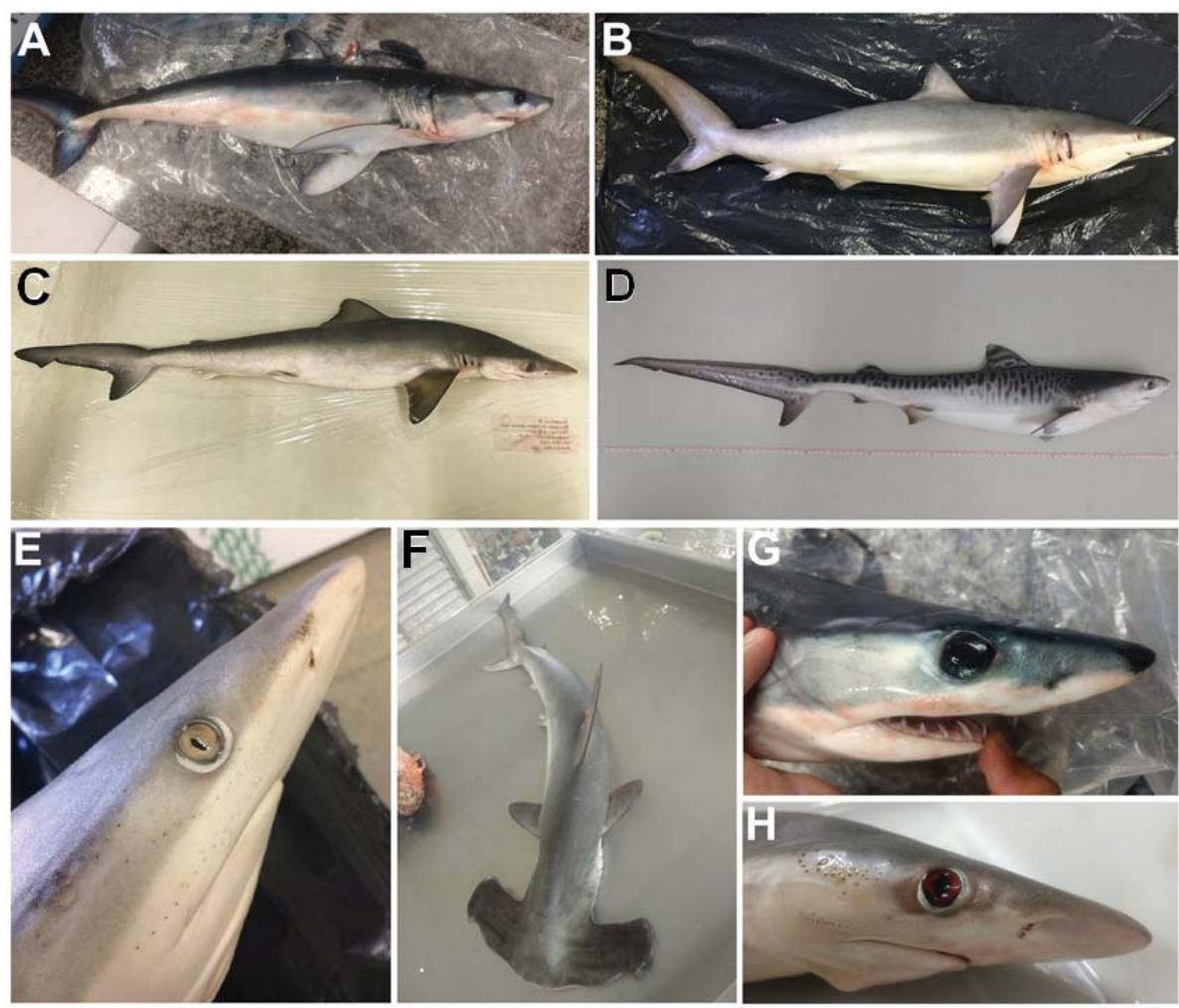

Figure 4. Shark species identified during this study. (A) Isurus oxyrinchus; (B) Carcharhinus brevipinna; (C) Rhizoprionodon lalandii; (D) Galeocerdo cuvier; (E) Eye detail - Carcharhinus brevipinna; (F) Sphyrna lewini.; (G) Eye detail - Isurus oxyrinchus; (H) Eye detail Rhizoprionodon lalandii. 
Figure 3 illustrates elasmobranch landings and weighing by artisanal fishers for marketing, while Figures 4 and 5 illustrate some of the rays and sharks, respectively, identified during this study.

\section{DISCUSSION}

The present study reports a preliminary assessment carried out during three years regarding artisanal fishing elasmobranch landing at three locations throughout the coast of Rio de Janeiro. Although the Associação de Pesca de Tamoios, in Cabo Frio is home to the previously described upwelling phenomenon, the highest richness concerning oceanic elasmobranchs was recorded at the Z-13 colony, in Copacabana, located in the metropolitan region of Rio de Janeiro. This sampling site is a part of Guanabara Bay, and the results presented herein reinforce the significant biodiversity of the bay and the importance of its recovery for elasmobranch management and conservation strategies.

In addition, many specimens belonging to different species sampled from this site (Sphyrna zygaena, Sphyrna lewini, Carcharinus brevipinna, Isurus oxyrinchus and Galeocerdo cuvier) were juvenile (Araujo, pers. obs.), while three spinner shark females (Carcharinus brevipinna) with mature oocytes were also noted (Araujo, pers. obs.), indicating that this may be a strategic reproduction and juvenile settlement site for several of the landed species. This is further supported by the record of six females of Rhizoprionodon lalandii, a coastal species, containing embryos and/or oocytes, as well as the high presence of juvenile hammerhead sharks (Sphyrna lewini), indicating potential development area (Motta et al., 2005). It is noteworthy both Itaipu and Copacabana are inserted in the estuarine Guanabara Bay, while Tamoios is located the São João River, both a strong indication of an elasmobranch breeding area, as reported by Plumlee et al. (2018) and Parsons and Hoffmayer (2007). In addition to species that are more commonly caught by gillnets, more pelagic and cosmopolitan species such as Tiger (Galeocerdo cuvier) and Mako (Isurus oxyrinchus) sharks were also caught by the same fishing methodology, all juveniles, once again indicating potential elasmobranch breeding and development areas.

In its most recent report, the Rio de Janeiro Institute for Fisheries Foundation (FIPERJ) reported that, in 2016, the species identified as cação-frango (Sharpnose shark, Rhizoprionodon sp.) was the most representative species regarding total landings, with a production of 5,212,8 kg, followed by cação-anjo (angel shark, Squatina sp.), at 2,661.5 kg and, finally, cação-martelo (hammerhead shark, Sphyrna sp.), totaling $1,004 \mathrm{kgs}$ (Fundação Instituto de Pesca do Estado do Rio de Janeiro 2016). However, the data reported herein cannot be quantitatively compared to FIPERJ data, as FIPERJ provides mixed and generalized information containing artisanal, semiindustrial and industrial fishing data, where artisanal fishing represents only a portion of the catch. In addition, the different range of fishing fleets should also be taken into account, as a limiting factor for the habitat range of certain species, such as hammerhead sharks and angelfish, due to depths and distance from the shore. It is also noteworthy that FIPERJ accounts for the weight of a certain species and data per individual, which may overestimate the data, as an adult fish may weigh more than 10 juvenile individuals.

Concerning species vulnerability, Squatina occulta and Squatina guggenheim, are classified as critically endangered and endangered, respectively, according to the IUCN. In addition, Squatina guggenheim, was reported as reaching critically low levels and populational declines during the 1990s (Boeckman \& Vooren, 1997; Vooren 1997; Lessa et al. 1999), indicating significant vulnerability for this species. In turn, all six hammerhead shark species distributed along the Brazilian coast are currently nationally listed as threatened (Instituto Chico Mendes de Conservação da Biodiversidade 2018). Given that the present study identified several Squatina guggenheim, Sphyrna lewini and Sphyrna zygaena individuals, it is clear that environmental education actions, measures to avoid the capture of this species or release actions alongside fishers at these locations are required, in order to sensitize them to the inherent impact of capturing these species and avoid further damage.

A significant amount of landed elasmobranch species is unidentified, or identified solely by their common names by the fishers, which also change according to fishery region (FIPERJ, 2016). For example, many unidentified species may belong to the cação category, the Brazilian generic name for most shark species, representing a significant amount of the 3.1 tons landed in 2016 for which no scientific identification was carried out. The same is probably true for rays, as these animals are distributed into categories with popular names such as raia-manteiga (stingrays) (about 1 ton) or raia-pintada (spotted rays) (3 tons) (FIPERJ, 2016). Thus, many species may be easily confused, making it difficult to create an elasmobranch management plan, and species identification is paramount. In addition, it is important to note the significant elasmobranch role as a fishing resource in Brazil, attributed to the current market incentive for the consumption of shark meat, due to, mainly, low costs, leading to social and economic, 
as well as ecological, concerns (Bornatowski et al. 2018).

Rio de Janeiro extractive marine fisheries represent about $2.5 \%$ of total shark landings caught per year in Brazil (Ministério da Pesca e Aquicultura 2014). However, in 2010 alone, in the Southwest Atlantic region, about 33\% more sharks were fished in reality compared to lower figures reported by Southwest Atlantic governments to the Food and Agriculture Organization of the United Nations (FAO), attributed to artisanal, illegal and discarded fisheries (Pauly \& Zeller, 2016). Allied to the lack of knowledge on the commercial and artisanal exploitation of these animals, the lack of information on the occurrence of shark species makes it difficult to employ conservation strategies aiming at the preservation and management of this resource. One way to circumvent the reported difficulties is the scientific monitoring of the artisanal fishermen colonies and performing a scientific approach towards the ecological knowledge of these fishermen, which, besides contributing to landing monitoring, enables taxonomic, physiological and genetic elasmobranch studies, furthering knowledge concerning the population ecology and geographical distribution of the group. In recent years, several studies have been conducted in Brazil addressing these issues (Palmeira et al. 2013; Barbosa-Filho et al. 2014, 2016, 2017; Bornatowski et al. 2015; Gemaque et al. 2017; Feitosa et al. 2018), denoting the relevance of information of this nature to the management and conservation of this threatened zoological group.

Dulvy et al. (2014) estimated that about a quarter of the world's shark and ray species are threatened by overfishing, and although the state of Rio de Janeiro is flagged as one of the world's priority shark conservation areas (Lucifora et al. 2011), the results presented herein indicate high amounts of threatened species being caught as by-catch. From an ecosystem perspective, it is well known that the presence of top predators and mesopredators as trophic regulators is essential for the functioning of ecosystem interaction webs (Stevens et al. 2000). Thus, declines in shark and ray population stocks may lead detrimental effects on marine communities (Myers et al. 2007; Bornatowski et al. 2014). The main documented phenomenon implies in the deregulation of lower trophic level maintenance exerted by top predator pressure, known as the topdown effect, thus affecting not only the ecosystem in question, but also the fishing industry itself (Ferretti et al. 2010).

In this regard, it is important to take into account the presence of keystone species. Keystone species are defined as species which "have an extremely high impact on a particular ecosystem relative to its population, and are also critical for the overall structure and function of an ecosystem, and influence which other types of organisms make up that ecosystem" (Humphries et al., 2017). Six keystone elasmobranch species have been previously observed throughout the Southeastern coast of Brazil (Bornatowski et al. 2014), and the present study reports four of them, namely Galeocerdo cuvier, Sphyrna lewini, S. zygaena and Zapteryx brevirostris, further indicating the importance of future assessments concerning elasmobranch fisheries monitoring in Brazil. In addition, due to the continuity of oceanographic conditions between the Brazilian Southeast and South, it is probable that the species reported herein display an extended ecosystem importance to the South, implicating in further social and economic concerns.

\section{ACKNOWLEDGEMENTS}

The authors would like to thank Ulisses L. Gomes for identifying the specimens deposited at the UERJ ichhtyological collection, Sérgio C. Moreira for kindly preparing the map and Marcelo Alves da Purificação, FIPERJ/Tamoios, Cabo Frio, for his help during field trips and data collection. In addition, special thanks are due to all fishers who aided in elasmobranch sampling and data collection, especially Manasi Rebouças and Manel Rebouças from the Z-13 Copacabana colony for their invaluable help and friendship. NLFA would like to thank $\mathrm{CNPq}$ for financial support in the form of an undergraduate scholarship, while CAL and VBB thank CAPES for financial support in the form of graduate scholarships. This study was financed in part by the Coordenação de Aperfeiçoamento de Pessoal de Nível Superior - Brasil (CAPES) Finance Code 001. This is a Programa Fiocruz de Fomento à Inovação (INOVA): Elasmobrânquios como ferramentas bioindicadoras de contaminação por metais no Sudeste Brasileiro: Impactos na saúde pública e no contexto socioambiental de populações vulneráveis locais (VPPIS-004-FIO-18) contribution. INOVA supports RAHD and SS.

\section{REFERENCES}

BARBOSA-FILHO, M.L.V., SCHIAVETTI, A., ALARCON, D.T., COSTA-NETO, E.M. 2014. "Shark is the man!": Ethno knowledge of Brazil's South Bahia fishermen regarding shark behaviors. $J$. Ethnobiol. Ethnomed.10: 54.

BARBOSA-FILHO, M.L.V., SICILIANO, S., COSTA-NETO, E.M. 2017. Knowledge and Practices of Expert Fishermen of South Bahia, Brazil, Regarding the International Shark Fin Market. Hum. 
Ecol., 45(1): 67-75.

BARBOSA-FILHO, M.L.V., TAVARES, D.C., SICILIANO, S., MOURA, J.F., COSTA-NETO, E.M., MOTTA, F.S., KOIKE, C.D.V. 2016. Interactions between whale sharks, Rhincodon typus Smith, 1928 (Orectolobiformes, Rhincodontidae), and Brazilian fisheries: The need for effective conservation measures. Mar. Pol., 73: 210-215.

BORNATOWSKI, H., BRAGA, R.R. \& BARRETO, R.P. 2017. Elasmobranch consumption in Brazil: Impacts and consequences In: ROSSI-SANTOS, M.R. \& FINKL, C.W. (Org.) Advances in Marine Vertebrate Research in Latin America. Coastal Research Library, $508 \mathrm{p}$.

BORNATOWSKI, H., BRAGA, R.R. \& VITULE, J.R.S. 2013. Shark mislabeling threatens biodiversity. Science., 340: 923.

BORNATOWSKI, H., BRAGA, R.R., KALINOWSKI, C. \& VITULE, J.R.S. 2015. "Buying a Pig in a Poke": the problem of Elasmobranch meat consumption in Southern Brazil. Ethnobiol. Lett. 6(1): 196-202.

BORNATOWSKI, H., NAVIA, A.F., BRAGA, R.R., ABILHOA, V. \& CORRÊA, M.F.M. 2014. Ecological importance of sharks and rays in a structural foodweb analysis in southern Brazil. ICES J. Mar. Sci., 71(7): 1586-1592.

BRITO, V. B. 2016. Etnoecologia da pesca de tubarões na Praia de Itaipu - RJ. Monograph, Bachelor in Environmental Sciences, Department of Marine Ecology and Resources, Federal University of the state of Rio de Janeiro, 44p.

COSTA, M.D.P., MILLS, M., RICHARDSON, A.J., FUlLER, R.A., MUELBERT, J.H. \& POSSINGHAM, H.P. 2018. Efficiently enforcing artisanal fisheries to protect estuarine biodiversity. Ecol. Appl., 28(6): 1450-1458.

DENT, F. \& CLARKE, S. 2015. State of the global market for shark products. Rome: Food \& Agriculture Organization of the United Nations (FAO). 187 p.

DULVY, N.K., HARISSON, L.R., CARLSON, J.K., CAVANAGH, R.D., KYNE, P.M., HARRISON, L.R. et al. 2014. Extinction risk and conservation of the world's sharks and rays. Elife., 3:e00590.

FEITOSA, L.M., MARTINS, A.P.B., GIARRIZZO, T., MACEDO, W., MONTEIRO, I. L., GEMAQUE, R. et al. 2018. DNA-based identification reveals illegal trade of threatened shark species in a global elasmobranch conservation hotspot. Sci. Rep., 8: 33-47.

FERRETTE, B.P.S., DOMINGUES, R.R., ROTUNDO, M.M., MIRANDA, M.P., BUNHOLI,
I.V., DE BIASI, J.B. et al. 2019. DNA Barcode Reveals the Bycatch of Endangered Batoids Species in the Southwest Atlantic: Implications for Sustainable Fisheries Management and Conservation Efforts. Genes., 10(304): 1-15.

FERRETTI, F., WORM, B., BRITTEN, G.L., HEITHAUS, M.R. \& LOTZE, H.K. 2019. Patterns and ecosystem consequences of shark declines in the ocean. Ecol. Lett., 13: 1055-1071.

FIPERJ. Entidades do Setor. Colônia. Rio de Janeiro: Fundação Instituto de Pesca do Estado do Rio de Janeiro, [2019]. Available at: <http://www.fiperj. rj.gov.br/index.php/entidade>.

FIPERJ. Relatório 2015. Disponível em: http:// www.fiperj.rj.gov.br/fiperj_imagens/arquivos/ revistarelatorios2015.pdf

FIPERJ. Relatório 2016. Disponível em: http:// www.fiperj.rj.gov.br/fiperj_imagens/arquivos/ revistarelatorios2015.pdf

GEMAQUE, R., MONTEIRO, I.L.P., GOMES, F., SODRE, D., SAMPAIO, I., SALES, J.B.L. et al. 2013. Why implement measures to conserve the diversity of Elasmobranchs? The case of the northern coast of Brazil. Rev. Biol., 17(2): 1-7.

GOMES, U.L., SIGNORI, C., GADIG, O.B.F. \& SANTOS, H.R.S. 2010. Guia para identificação de tubarões e raias do Rio de Janeiro. Rio de Janeiro: Technical Books. $234 \mathrm{p}$.

HEUPEL, M.R., KNIP, D.M., SIMPFENDORFER, C.A. \& DULVY, N.K. 2014. Sizing up the ecological role of sharks as predators. Mar. Ecol. Prog. Ser., 495: 291-298.

HUMPRHIES, M.M., STUDD, E.K., MENZIES, A.K. \& BOUTIN, S. To everything there is a season: Summer-to-winter food webs and the functional traits of keystone species. Integr. Comp. Biol. 57: 961-967.

IBGE. 2019. Disponível em: www.ibge.gov.br

INSTITUTOCHICOMENDESDECONSERVAÇÃO DA BIODIVERSIDADE. 2011. BOLETIM ESTATÍSTICO DA PESCA E AQUICULTURA. Disponível em: http://www.icmbio.gov.br/cepsul/ images/stories/biblioteca/download/estatistica/ est_2011_bol_bra.pdf

INSTITUTOCHICOMENDES DECONSERVAÇÃO DA BIODIVERSIDADE. 2018. Livro Vermelho da Fauna Brasileira Ameaçada de Extinção: Volume VI - Peixes. In: INSTITUTO CHICO MENDES DE CONSERVAÇÃO DA BIODIVERSIDADE (Org.). Livro Vermelho da Fauna Brasileira Ameaçada de Extinção. Brasília: ICMBio. 1232 p.

KALILOSKI, D.C. \& VASCONCELLOS, M. 2012. Case study of the technical, socio-economic and 
environmental conditions of small-scale fisheries in the estuary of Patos Lagoon, Brazil: a methodology for assessment. Rome: Food \& Agriculture Organization of the United Nations (FAO). 224 p.

KOLDING, J., BENÉ, C. \& BAVINCK, M. 2010. Small-scale fisheries: Importance, vulnerability and deficient knowledge. In: GARCIA, S. M., RICE, J., \& CHARLES, A. (Org.). (2014). Governance of Marine Fisheries and Biodiversity Conservation, Interaction and coevolution. Wiley-Blackwell. pp. 1-21.

LACK, M. \& SANT, G. 2009. Trends in Global Shark Catch and Recent Developments in Management. Cambridge: TRAFFIC International. 29 p.

LESSA, R., SANTANA, F.M., RINCON, G., GADIG, O.B.F. \& EL-DEIR, A.C.A. 1999. Biodiversidade de Elasmobrânquios do Brasil. Recife: Ministério do Meio Ambiente. 91 p.

LUCIFORA, L., GARCÍA, V. \& WORM, B. 2011. Global diversity hotspots and conservation priorities for sharks. PloS One., 6(5): 1-7.

MACE, G.M., COLLAR, N.J., GASTON, K.J., HILTON-TAYLOR, C., AKCAKAYA, H.R., LEADER-WILLIAMS, N., MILNER- GULLAND, E.J. \& STUART SN. 2008. Quantification of extinction risk: IUCN's system for classifying threatened species. Conserv. Biol., 22: 1424-1442.

MENDONÇA, F.F., HASHIMOTO, D. T., PORTOFORESTI, F., OLIVEIRA, C., GADIG, O.B.F. \& FORESTI, F. 2019. Identification of the shark species Rhizoprionodon lalandii and $R$. porosus (Elasmobranchii, Carcharhinidae) by multiplex PCR and PCR-RFLP techniques. Mol. Ecol. Resour., 9: 771-773.

MINISTÉRIO DA PESCA E AQUICULTURA. 2014. Pesca Artesanal. Brasília: Ministério da Pesca e Aquicultura, 2014. Disponível em: <http://www. mpa.gov.br/pesca/artesanal $>$.

MOLINA, J.M. \& COOKE, S.J. 2012. Trends in shark bycatch research: current status and research needs. Rev. Fish. Biol. Fisheries., 22(3): 719-737.

MOTTA, F.S., GADIG, O.B., NAMORA, R.C. \& BRAGA, F.M., 2005. Size and sex compositions, length-weight relationship, and occurrence of the Brazilian sharpnose shark, Rhizoprionodon lalandii, caught by artisanal fishery from southeastern Brazil. Fish. Res. 74: 116-126.

MYERS, R.A., BAUM, J.K., SHEPHERD, T.D., POWERS, S.P. \& PETERSON, C.H. 2007. Cascading effects of the loss of apex predatory sharks from a coastal ocean. Science., 315: 1846-1850.

NETTO, E.B.F., GALEZER, L.R. 1991. Associações de peixes bentônicas e demersais na região de Cabo Frio, RJ, Brasil. Nerítica, 6:139-156.

PALMEIRA, C.A.M., RODRIGUES-FILHO, L.F.S., SALES, J.B.L., VALLINOTO, M., SCHNEIDER, H. \& SAMPAIO, I. 2013. Commercialization of a critically endangered species (largetooth sawfish, Pristis perotteti) in fish markets of northern Brazil: Authenticity by DNA analysis. Food Control., 34: 249-252.

PARSONS, G.R. \& HOFFMAYER, E.R., 2007. Identification and characterization of shark nursery grounds along the Mississippi and Alabama gulf coasts, American Fisheries Society Symposium. American Fisheries Society, p. 301.

PAULY, D. \& ZELLER, D. 2016. Catch reconstructions reveal that global marine fisheries catches are higher than reported and declining. Nat. Commun., 7(10244): $1-9$.

PLUMLEE, J.D., DANCE, K.M., MATICH, P., MOHAN, J.A., RICHARDS, T.M., TINHAN, T.C., FISHER, M.R. \& WELLS, R.D., 2018. Community structure of elasmobranchs in estuaries along the northwest Gulf of Mexico. Estuar. Coast Shelf S., 204: 103-113.

PREVIERO, M. \& GASALLA, M.A. 2018. Mapping fishing grounds, resource and fleet patterns to enhance management units in data-poor fisheries: The case of snappers and groupers in the Abrolhos Bank coralreefs (South Atlantic). Ocean Coast Manag., 154: 83-95.

STEVENS, J.D.R., BONFIL, R., DULVY, N.K. \& WALKER, P.A. 2000. The effects of fishing on sharks, rays, and chimaeras (Chondrichthyans), and the implications for marine ecosystem. ICES J. Mar. Sci., 57: 476-494.

VALENTIN, J. L. 2001. The Cabo Frio Upwelling System, Brazil. In: SEELIGER, U. 7 KJERFVE, B. (Org.) Coastal Marine Ecosystems of Latin America. Berlin: Springer-Verlag Berlin Heidelberg. p. 97-105.

VANNUCCINI, S. 1999. Shark utilization, market and trade. Rome: Food \& Agriculture Organization of the United Nations (FAO). $470 \mathrm{p}$.

VASCONCELOS, M., DIEGUES, A.C. \& SALES, R.R. 2007. Limites e possibilidades na gestão da pesca artesanal costeira. In: COSTA, A.L. (Org.) Nas Redes da Pesca Artesanal. Brasília: IBAMA-MMA. pp.15-83.

VOOREN, C.M. Demersal elasmobranchs. In: SEELIGER, U., ODEBRECHT, C. \& CASTELLO, J.P. (Ed.). Subtropical convergence environment: the coast and the sea in the Southwestern Atlantic. Berlin: Springer. p. 41-146. 